\title{
Inverse Problems for a Generalized Subdiffusion Equation with Final Overdetermination
}

\author{
Nataliia Kinash and Jaan Janno \\ Department of Cybernetics, Tallinn University of Technology \\ Ehitajate tee 5,19086 Tallinn, Estonia \\ E-mail: nataliia.kinash@taltech.ee \\ E-mail(corresp.): jaan.janno@taltech.ee
}

Received November 22, 2019; revised February 4, 2019; accepted February 6, 2019

\begin{abstract}
We consider two inverse problems for a generalized subdiffusion equation that use the final overdetermination condition. Firstly, we study a problem of reconstruction of a specific space-dependent component in a source term. We prove existence, uniqueness and stability of the solution to this problem. Based on these results, we consider an inverse problem of identification of a space-dependent coefficient of a linear reaction term. We prove the uniqueness and local existence and stability of the solution to this problem.
\end{abstract}

Keywords: inverse problem, subdiffusion, final overdetermination, fractional diffusion.

AMS Subject Classification: 35R30; 35R11.

\section{Introduction}

Anomalous diffusion processes are described by different models [6]. Among them stands out the time (or space-time) fractional diffusion equation that is the most common way to represent a subdiffusion. For some situations such approach does not work [19]. Therefore, more general models that unify wider range of subdiffusion processes are introduced $[19,25]$.

In this paper we use an operator that is more general than the fractional time derivative:

$$
D_{t}^{\{k\}} v=\frac{d}{d t} k * v
$$

where $*$ denotes the time convolution, i.e. $\left(v_{1} * v_{2}\right)(t)=\int_{0}^{t} v_{1}(t-\tau) v_{2}(\tau) d \tau$. Taken $k=\frac{t^{-\beta}}{\Gamma(1-\beta)},(1.1)$ transforms into a well-known Riemann-Liouville frac- 
tional derivative. The operator corresponding to the Caputo fractional derivative denoted as $\mathbb{D}_{(k)}^{C} v$ was introduced by Luchko and Yamamoto in [23] and also in [15].

The toolkit for treating such a type of derivative have been developed by Prüss et al. [5]. They have created a setting to introduce the operator inverse to $D_{t}^{\{k\}}$ through the concept of Completely Positive kernels [5]: a kernel $M \in$ $L_{1, \text { loc }}\left(\mathbb{R}_{+}\right)$is called completely positive if there are $k_{0} \geq 0$ and nonnegative and nonincreasing $k_{1} \in L_{1, l o c}\left(\mathbb{R}_{+}\right)$such that $M *\left(k_{0} \delta+k_{1}\right)=1$ holds. The applications of this concept can be found in $[1,33,34]$. Another approach to this issue has been developed by Kochubei [19].

Often parameters of models are unknown. Then additional observations are performed and inverse problems solved to reconstruct unknown quantities $[12,13,16,17,20,21]$. In the present paper we consider two inverse problems (IPs) that use final observation data: IP1 is to identify a space-dependent factor $f$ of a source term $g(t, x) f(x)$; IP2 is to reconstruct a coefficient $r(x)$ of a linear reaction term.

IP1 for fractional and perturbed fractional diffusion equations is studied in several papers. Theoretical and numerical results are obtained in the particular case $g=g(t)[7,17,18,26]$ and in the case $g=g(t, x)[30,32]$. In latter papers the existence and uniqueness of solutions are proved for almost all scalar diffusion coefficients. IP1 for a semilinear fractional diffusion equation is considered in [15]. Uniqueness of the solution is proved.

In this paper we consider IP1 for a more general diffusion equation that includes the operator (1.1) instead of the fractional derivative. We prove the uniqueness of the solution to IP1 by applying a modified version of the positivity principle from [15]. That falls into category of maximum principle results $[13,20,22]$. Similar approaches to the inverse problems are well-known in the domain of parabolic equations $[2,12]$. Next we prove the existence and stability of the solution of IP1 by means of the Fredholm alternative. The uniqueness of solution of IP2 follows from the IP1-results. Finally, we prove local existence and stability of the solution to IP2 by means of the contraction argument.

\section{Formulation of direct and inverse problems}

Let us consider the generalized subdiffusion equation

$$
U_{t}(t, x)=(M * L U)_{t}(t, x)+Q(t, x)
$$

where $U$ physical state, $t$ is the time, $x \in \mathbb{R}^{n}$ is a space variable, $Q$ is a source term, the operator $L=L(x)$ is such that

$L(x)=L_{1}(x)+r(x) I$, where $L_{1}(x)=\sum_{i, j=1}^{n} a_{i j}(x) \frac{\partial^{2}}{\partial x_{i} \partial x_{j}}+\sum_{j=1}^{n} a_{j}(x) \frac{\partial}{\partial x_{j}}$

and $I$ is the unity operator. The kernel $M$ is a memory function related to a non-locality of the diffusion process. 
There are two ways to derive the equation (2.1) from physical laws. One method consists in modelling continuous time random walk processes in microlevel and taking a continuous limit in a macro-level [4] and another one uses conservative laws and specific constitutive relations with memory [27].

Real world applications of the equation (2.1) include diffusion in fractal and porous media, e.g. propagation of pollution, heat flow in media with memory, dynamics of protein in cells, transport in dielectrics and semiconductors, usage of optical tweezers, Hamiltonian chaos etc. [3, 4,6, 27,31].

Let us assume that there is a function $k$ such that $k * M=M * k=1$. Then if we apply $k *$ to (2.1), we obtain an equation that contains the explicit differential operator $L$ and is called the normal form of $(2.1): k * U_{t}(t, x)=$ $L U(t, x)+H(t, x)$, where $H(x, t):=k * Q(t, x)$. The term $k * U_{t}$ can be rewritten in the form $D_{t}^{\{k\}}(U-U(0, \cdot))$ that does not contain the 1st order derivative of $U$. Therefore, we get the equation

$$
D_{t}^{\{k\}}(U-U(0, \cdot))=L U(t, x)+H(t, x) .
$$

Conversely, in case of sufficiently regular $U$, the equation (2.1) follows from (2.2) by means of the application of the operator $\frac{\partial}{\partial t} M *$.

The equation (2.1) and its analogue (2.2) incorporate the following possibilities:

1. The kernel $M(t)=\frac{t^{\beta-1}}{\Gamma(\beta)}, 0<\beta<1$, represents a power-type memory. Then (2.1) becomes the celebrated time fractional diffusion equation $U_{t}=\varkappa D^{1-\beta} L U+Q$, where $D^{1-\beta} v=\left(\frac{t^{\beta-1}}{\Gamma(\beta)} * v\right)_{t}$ is the RiemannLiouville fractional derivative of the order $1-\beta[4,17,20,26]$. For such $M$, it holds $k=\frac{t^{-\beta}}{\Gamma(1-\beta)}$ and $\left[k *(v-v(0)]_{t}=k * v_{t}=\partial_{t}^{\beta} v\right.$ is the Caputo fractional derivative.

2. The kernel $M$ or its associate $k$ is a linear combination of power functions $[25,31]$ :

$$
\begin{aligned}
& M(t)=\frac{t^{\beta-1}}{\Gamma(\beta)}+\sum_{j=1}^{l} p_{j} \frac{t^{\beta_{j}-1}}{\Gamma\left(\beta_{j}\right)}, \quad 0<\beta<\beta_{j}<1, \quad p_{j} \geq 0, \\
& k(t)=\frac{t^{-\beta}}{\Gamma(1-\beta)}+\sum_{j=1}^{l} q_{j} \frac{t^{-\beta_{j}}}{\Gamma\left(1-\beta_{j}\right)}, \quad 0<\beta_{j}<\beta<1, \quad q_{j} \geq 0 .
\end{aligned}
$$

3. The kernel $M$ has the form $M(t)=\int_{0}^{1} p(s) \frac{t^{s-1}}{\Gamma(s)} d s$ where $p \geq 0$ is a nonvanishing integrable function (cf. $[3,25,31]$ ). Such a kernel stands for the distributed order fractional derivative that is used for modeling diffusion with a logarithmic growth of the mean square displacement [19].

4. Tempered fractional calculus [29], that is another way to generalize a fractional calculus, falls into the case

$$
M(t)=\frac{1}{\Gamma(\beta)} e^{-\lambda t} t^{\beta-1}+\frac{\lambda}{\Gamma(\beta)} \int_{0}^{t} e^{-\lambda \tau} \tau^{\beta-1} d \tau, \quad \lambda>0 .
$$


This type of kernel is used for modelling the transition from anomalous to normal diffusion.

Every presented example of $M$ (or $k$ ) has a completely monotonic associate $k$ (or $M$ ) that solves $k * M=1$ (see Section 3).

Let $\Omega \in \mathbb{R}^{n}$ be an open bounded domain with the boundary $\partial \Omega$. In direct problem we have to find a function $u$ that solves the initial-boundary value problem

$$
\begin{aligned}
& D_{t}^{\{k\}}(U-\Phi)(t, x)=L U(t, x)+H(t, x), \quad x \in \Omega, t \in(0, T), \\
& U(0, x)=\Phi(x), \quad x \in \Omega, \\
& \mathcal{B}(U-b)(t, x)=0, \quad x \in \partial \Omega, t \in(0, T) .
\end{aligned}
$$

Here $\Phi$ and $b$ are given functions and

$$
\mathcal{B} v(x)=v(x) \quad \text { or } \quad \mathcal{B} v(x)=\omega(x) \cdot \nabla v(x),
$$

with $\omega \cdot \nu>0$ and $\nu(x)$ denoting the outer normal of $\partial \Omega$ at $x \in \Omega$. An important particular case is $\omega=\left(\left.\sum_{j=1}^{n} a_{i j} \nu_{j}\right|_{i=1, \ldots, n}\right)$. Then the condition $\left.\mathcal{B}(U-b)\right|_{(t, x) \in(0, T) \times \partial \Omega}=0$ corresponds to the flux specified at $\partial \Omega$.

Let us proceed to inverse problems. To this end we introduce the condition

$$
U(T, x)=\Psi(x), \quad x \in \Omega,
$$

with a given observation function $\Psi$. Firstly, we formulate of an inverse source problem. Let

$$
H(t, x)=g(t, x) f(x)+h_{0}(t, x),
$$

where the components $g f$ and $h_{0}$ may correspond to different sources or sinks. The factor $f$ is unknown and to be reconstructed by means of the data (2.4). Since the whole function $U$ is also unknown, the first inverse problem consists in determination a pair of functions $(f, U)$ that satisfies $(2.3),(2.4)$ and $(2.5)$.

In the second inverse problem, our aim is to identify the coefficient $r$ of the linear reaction term $r U$. In the mathematical formulation, the problem consists in finding a pair $(r, U)$ that satisfies $(2.3)$ and $(2.4)$. We can handle the case of zero initial condition $\Phi=0$ (for details, see the end of Section 6).

Methods to be used in this paper require homogeneous boundary conditions. Therefore, we perform the change of the second unknown $u=U-b$ in our problems. It brings along shifts of data by addends containing $b$.

Firstly, from (2.3) we obtain the following problem for $u=U-b$ :

$$
\begin{aligned}
& D_{t}^{\{k\}}(u-\varphi)(t, x)=L u(t, x)+F(t, x), \quad x \in \Omega, t \in(0, T), \\
& u(0, x)=\varphi(x), \quad x \in \Omega \\
& \mathcal{B} u(t, x)=0, \quad x \in \partial \Omega, t \in(0, T),
\end{aligned}
$$

where

$$
\begin{aligned}
& \varphi(x)=\Phi(x)-b(0, x) \\
& F(t, x)=H(t, x)+L b(t, x)-D_{t}^{\{k\}}(b-b(0, \cdot))(t, x) .
\end{aligned}
$$


The overdetermination condition (2.4) in terms of $u$ has the form

$$
u(T, x)=\psi(x), \quad x \in \Omega,
$$

where $\psi(x)=\Psi(x)-b(T, x)$. Plugging (2.5) into (2.8) we obtain

$$
F(t, x)=g(t, x) f(x)+h(t, x),
$$

where $h(t, x)=h_{0}(t, x)+L b(t, x)-D_{t}^{\{k\}}(b-b(0, \cdot))(t, x)$.

In the reformulated first inverse problem (IP1), we seek for the pair of functions $(f, u)$ that satisfies $(2.6),(2.9)$ and $(2.10)$.

Let us reformulate the second inverse problem, too. From the relations (2.3), (2.4) with $\Phi=0$ by means of the change of variable $u=U-b$, we obtain the following problem for the pair $(r, u)$ :

$$
\begin{aligned}
& D_{t}^{\{k\}} u(t, x)=L_{1} u(t, x)+r(x)(u+b)(t, x)+F_{1}(t, x) \quad x \in \Omega, t \in(0, T), \\
& u(0, x)=0, \quad x \in \Omega, \quad \mathcal{B} u(t, x)=0, \quad x \in \partial \Omega, t \in(0, T), \\
& u(T, x)=\psi(x), \quad x \in \Omega,
\end{aligned}
$$

where $b(0, x)=0, x \in \Omega$, the function $\psi$ is expressed by $\psi(x)=\Psi(x)-b(T, x)$ and $F_{1}(t, x)=H(t, x)+L_{1} b(t, x)-D_{t}^{\{k\}} b(t, x)$.

Thus, the reformulated second inverse problem (IP2) is to find the pair of functions $(r, u)$ that satisfies $(2.11)$.

\section{Basic assumptions}

In this section we collect basic conditions on the domain, operator $L$ and kernels $k$ and $M$ that will be assumed throughout the paper.

We assume that $\partial \Omega$ is uniformly of the class $C^{2}$ and $\omega \in\left(C^{1}(\partial \Omega)\right)^{n}$. Moreover, we assume that $a_{i j}, a_{j}, r \in C(\bar{\Omega})$ and the principal part of $L$ is uniformly elliptic, i.e. $\sum_{i, j=1}^{n} a_{i j}(x) \xi_{i} \xi_{j} \geq c|\xi|^{2} \forall \xi \in \mathbb{R}^{n}, x \in \Omega$ for some $c>0$.

Concerning the function $k$, we assume that

1. $k$ belongs to $L_{1, l o c}(0, \infty)$ and is a solution of the equation $M * k=1$ with a kernel $M \in L_{1, l o c}(0, \infty)$ that satisfies the conditions

$$
\begin{aligned}
& M \in C^{1}(0, \infty), \quad \lim _{t \rightarrow 0^{+}} M(t)=\infty, \quad M>0, \quad M^{\prime} \leq 0, \\
& -M^{\prime} \text { is nonincreasing and convex; }
\end{aligned}
$$

2. $k$ has the following properties:

$$
\begin{aligned}
& k \in C(0, \infty), \quad \lim _{t \rightarrow 0^{+}} k(t)=\infty, \quad k>0, \quad k \text { is nonincreasing, } \\
& \exists t_{k}>0: k(t) \text { is strictly decreasing in }\left(0, t_{k}\right) .
\end{aligned}
$$


The assumptions (3.1) ensure the existence of a sufficiently regular solution of the direct problem (see Lemma 3) and the assumptions (3.2), (3.3) are needed for the application of a positivity principle to this solution.

We mention that restricting generality a bit it is possible to reduce all conditions 1 and 2 to the single kernel $M$. Firstly, $M \in L_{1, l o c}(0, \infty)$ and (3.1) imply the existence of a unique solution $k \in L_{1, l o c}(0, \infty)$ of the equation $k * M=1$ ( [10], Ch. 5, Corollary 5.6). Secondly, all properties (3.2), (3.3) follow from conditions that are a bit stronger than (3.1). It is shown in the following lemma. Proof is in Appendix.

Lemma 1. Let $M \in L_{1, l o c}(0, \infty)$ satisfy (3.1) and $M^{\prime}<0, \log M-$ convex, $\log \left(-M^{\prime}\right)$ - convex. Then the solution of $M * k=1$ satisfies (3.2), (3.3).

The imposed assumptions on $M$ and $k$ hold for weakly singular completely monotonic kernels from

$$
\mathcal{C M}=\left\{z \in L_{1, l o c}(0, \infty) \bigcap C^{\infty}(0, \infty): \lim _{t \rightarrow 0^{+}} z(t)=\infty,(-1)^{i} z^{(i)}>0, i=0,1, \ldots\right\} .
$$

For $M$ and $k$ satisfying $M * k=1$, it holds $M \in \mathcal{C M}$ if and only if $k \in \mathcal{C M}$ ( [9], Theorem 3).

All examples of $M$ and $k$ given in Section 2 belong to $\mathcal{C M}$.

\section{Preliminaries}

\subsection{Functional spaces}

Let $X$ be a Banach space. Since $k * M=1$, we have

$$
D_{t}^{\{k\}}(M * v)=\frac{d}{d t} k * M * v=\frac{d}{d t} 1 * v=v, \quad \forall v \in L_{1}((0, T) ; X),
$$

where $L_{1}((0, T) ; X)$ is the space of functions $u:(0, T) \rightarrow X$ that are integrable in the Bochner sense on $(0, T)$. This means that the operator $M *$ is a one-toone mapping from $L_{1}((0, T) ; X)$ to $\left\{M * v: v \in L_{1}((0, T) ; X)\right\}$ and $D_{t}^{\{k\}}$ is the inverse of $M *$.

As usual, let $C([0, T] ; X)$ stand for the Banach space of functions $u$ : $[0, T] \rightarrow X$ that are continuous on $[0, T]$ with the norm $\|u\|_{C([0, T] ; X)}=$ $\max _{t \in[0, T]}\|u(t)\|_{X}$ and $C_{0}([0, T] ; X)=\{u \in C([0, T] ; X): u(0)=0\}$. Based on the relation (4.1), we introduce the functional space

$$
C_{0}^{\{k\}}([0, T] ; X):=M * C([0, T] ; X)=\{M * v: v \in C([0, T] ; X)\} .
$$

It is a Banach space with the norm

$$
\|u\|_{C_{0}^{\{k\}}([0, T] ; X)}=\left\|D_{t}^{\{k\}} u\right\|_{C([0, T] ; X)} .
$$

Since $M * \in \mathcal{L}\left(C([0, T] ; X), C_{0}([0, T] ; X)\right)$, it holds

$$
C_{0}^{\{k\}}([0, T] ; X) \hookrightarrow C_{0}([0, T] ; X) .
$$


We also define the space

$$
\begin{aligned}
C^{\{k\}}([0, T] ; X):=C_{0}^{\{k\}}([0, T] ; X)+X \\
\quad=\left\{u: u(t)=u_{1}(t)+u_{2}, u_{1} \in C_{0}^{\{k\}}([0, T] ; X), u_{2} \in X\right\}
\end{aligned}
$$

that is a Banach space with the norm

$$
\|u\|_{C\{k\}([0, T] ; X)}=\|u-u(0)\|_{C_{0}^{\{k\}}([0, T] ; X)}+\|u(0)\|_{X} .
$$

Next we introduce the abstract Hölder spaces with corresponding norms

$$
\begin{aligned}
& C_{0}^{\alpha}([0, T] ; X)=\left\{u \in C_{0}([0, T] ; X):\right. \\
& \left.\qquad u \|_{C_{0}^{\alpha}([0, T] ; X)}:=\sup _{0<t_{1}<t_{2}<T} \frac{\left\|u\left(t_{2}\right)-u\left(t_{1}\right)\right\|_{X}}{\left(t_{2}-t_{1}\right)^{\alpha}}<\infty\right\}, \\
& C^{\alpha}([0, T] ; X)=C_{0}^{\alpha}([0, T] ; X)+X, \\
& \|u\|_{C^{\alpha}([0, T] ; X)}=\|u-u(0)\|_{C_{0}^{\alpha}([0, T] ; X)}+\|u(0)\|_{X},
\end{aligned}
$$

where $0<\alpha<1$, and define the Banach spaces with norms

$$
\begin{aligned}
& C_{0}^{\{k\}, \alpha}([0, T] ; X)=M * C_{0}^{\alpha}([0, T] ; X), \\
& \|u\|_{C_{0}^{\{k\}, \alpha}([0, T] ; X)}=\left\|D_{t}^{\{k\}} u\right\|_{C_{0}^{\alpha}([0, T] ; X)}, \\
& C^{\{k\}, \alpha}([0, T] ; X)=M * C^{\alpha}([0, T] ; X)+X, \\
& \|u\|_{C^{\{k\}, \alpha}([0, T] ; X)}=\left\|D_{t}^{\{k\}}(u-u(0))\right\|_{C^{\alpha}([0, T] ; X)}+\|u(0)\|_{X} .
\end{aligned}
$$

Let us establish some connections between the spaces (4.2), (4.3) and the usual $C, C^{1}$ - and Hölder spaces. For $C^{\{k\}}([0, T] ; X)$ the embeddings

$$
C^{1}([0, T] ; X) \hookrightarrow C^{\{k\}}([0, T] ; X) \hookrightarrow C([0, T] ; X)
$$

are valid. The right embedding follows from $M * \in \mathcal{L}(C([0, T] ; X))^{1}$. To prove the left embedding, we choose some $u \in C^{1}([0, T] ; X)$. Then

$\|u\|_{C\{k\}([0, T] ; X)}=\|u-u(0)\|_{C_{0}^{\{k\}}([0, T] ; X)}+\|u(0)\|_{X}=\left\|k * u^{\prime}\right\|_{C_{0}([0, T] ; X)}+\|u(0)\|_{X}$ and since $k * \in \mathcal{L}\left(C([0, T] ; X), C_{0}([0, T] ; X)\right)$, the left relation in (4.4) follows.

Analogous relations for the space $C_{0}^{\{k\}, \alpha}([0, T] ; X)$ are

$$
C_{0}^{1+\alpha}([0, T] ; X) \hookrightarrow C_{0}^{\{k\}, \alpha}([0, T] ; X) \hookrightarrow C_{0}^{\alpha}([0, T] ; X)
$$

where

$$
C_{0}^{1+\alpha}([0, T] ; X)=\left\{u: u, u^{\prime} \in C_{0}^{\alpha}([0, T] ; X)\right\} .
$$

The right embedding in (4.5) is a consequence of the fact that $M * \in$ $\mathcal{L}\left(C_{0}^{\alpha}([0, T] ; X)\right)$ (see Lemma 4.2 in $\left.[14]\right)$ and the left embedding in (4.5) can be proved similarly to the left embedding in (4.4).

Under additional assumptions on $M$ it is possible to show that the operator $M *$ increases the order of Hölder continuity of a function. Namely, the following lemma is valid. Its proof is deferred to Appendix.

\footnotetext{
1 The symbol $\mathcal{L}$ stands for the space of linear and bounded operators.
} 
Lemma 2. If $M(t) \leq c_{1} t^{\beta-1},\left|M^{\prime}(t)\right| \leq c_{2} t^{\beta-2}, t \in(0, T)$ for some $c_{1}, c_{2} \in$ $\mathbb{R}_{+}, 0<\beta \leq \alpha<1$ then $M * \in \mathcal{L}\left(C_{0}^{\alpha-\beta}([0, T] ; X), C_{0}^{\alpha}([0, T] ; X)\right)$.

Under conditions of Lemma $2, C_{0}^{\{k\}, \alpha-\beta}([0, T] ; X) \hookrightarrow C_{0}^{\alpha}([0, T] ; X)$. In the particular case $M(t)=\frac{t^{\beta-1}}{\Gamma(\beta)}$ (then $M *$ is the fractional integral of the order $\beta)$, it holds the equality $C_{0}^{\{k\}, \alpha-\beta}([0, T] ; X)=C_{0}^{\alpha}([0, T] ; X)[15]$.

By exchanging $M$ and $k$ in above relations, we obtain definitions and embeddings of spaces that contain $\{M\}$ instead of $\{k\}$ in the superscript.

\subsection{Abstract Cauchy problem}

Let $A: \mathcal{D}(A) \rightarrow X$ be a linear densely defined operator in a Banach space $X$. We say that $A$ belongs to the class $\mathcal{S}(\eta, \theta)$ for $\eta \in \mathbb{R}, \theta \in(0, \pi)$ if

$$
\begin{aligned}
& \rho(A) \supset \Sigma(\eta, \theta)=\{\lambda \in \mathbb{C}: \lambda \neq \eta, \arg |\lambda-\eta|<\theta\} \quad \text { and } \\
& \left\|(\mu-A)^{-1}\right\|_{\mathcal{L}(X)} \leq \frac{C}{|\mu-\eta|} \forall \mu \in \Sigma(\eta, \theta) \text { for some constant } C>0 .
\end{aligned}
$$

An operator $A \in \mathcal{S}(\eta, \theta)$ is closed. This implies that $X_{A}:=\mathcal{D}(A)$ is a Banach space with the graph norm $\|w\|_{X_{A}}=\|w\|_{X}+\|A w\|_{X}$.

Obviously, $\mathcal{S}\left(\eta, \theta_{1}\right) \subset \mathcal{S}\left(\eta, \theta_{2}\right)$ for $\theta_{1}>\theta_{2}$. Operators of the class $\mathcal{S}(\eta, \theta)$, $\theta \in\left(\frac{\pi}{2}, \pi\right)$, are the sectorial operators that generate analytic semigroups.

Now let us consider the Cauchy problem

$$
D_{t}^{\{k\}}(u-\varphi)(t)=A u(t)+F(t), \quad t \in[0, T], \quad u(0)=\varphi,
$$

with given $F:[0, T] \rightarrow X$ and $\varphi \in X$.

Lemma 3. Let $A \in \mathcal{S}\left(\eta, \frac{\pi}{2}\right)$ for some $\eta \in \mathbb{R}$. Then the following statements are valid.

(i) (uniqueness) Let $u \in C^{\{k\}}([0, T] ; X) \bigcap C\left([0, T] ; X_{A}\right)$ solve (4.6) and $\varphi=0$, $F=0$. Then $u=0$.

(ii) Let $F \in C_{0}^{\alpha}([0, T] ; X)$ and $\varphi=0$. Then (4.6) has a solution $u$ in the space $C_{0}^{\{k\}, \alpha}([0, T] ; X) \bigcap C_{0}^{\alpha}\left([0, T] ; X_{A}\right)$. This solution satisfies the estimate

$$
\|u\|_{C_{0}^{\{k\}, \alpha}([0, T] ; X) \cap C_{0}^{\alpha}\left([0, T] ; X_{A}\right)} \leq C_{1}\|F\|_{C_{0}^{\alpha}([0, T] ; X)} .
$$

(iii) Let $F \in C^{\alpha}([0, T] ; X)$ and $\varphi \in X_{A}$. Then (4.6) has a solution $u$ in the space $C^{\{k\}}([0, T] ; X) \bigcap C\left([0, T] ; X_{A}\right)$. This solution satisfies the estimate

$$
\|u\|_{C\{k\}([0, T] ; X) \cap C\left([0, T] ; X_{A}\right)} \leq C_{2}\left(\|F\|_{C^{\alpha}([0, T] ; X)}+\|\varphi\|_{X_{A}}\right) .
$$

The constants $C_{1}$ and $C_{2}$ depend on $M$ and $A$.

Proof. The change of variable $v=D_{t}^{\{k\}}(u-\varphi) \Leftrightarrow u=M * v+\varphi$ reduces (4.6) of the integral equation

$$
v(t)=A(M * v)(t)+F(t)+A \varphi, \quad t \in[0, T] .
$$


Provided $F \in C([0, T] ; X), \varphi \in X_{A}$, the function $u \in C^{\{k\}}([0, T] ; X) \cap$ $C\left([0, T] ; X_{A}\right)$ solves (4.6) if and only if $v \in V:=\{v \in C([0, T] ; X): M *$ $\left.v \in C_{0}\left([0, T] ; X_{A}\right)\right\}$ solves (4.9). Similar one-to-one correspondence holds for $u \in C_{0}^{\{k\}, \alpha}([0, T] ; X) \bigcap C_{0}^{\alpha}\left([0, T] ; X_{A}\right)$ and $v \in V^{\alpha}:=\left\{v \in C_{0}^{\alpha}([0, T] ; X):\right.$ $\left.M * v \in C_{0}^{\alpha}\left([0, T] ; X_{A}\right)\right\}$ in the particular case $F \in C_{0}^{\alpha}([0, T] ; X), \varphi=0$.

Since $M$ satisfies the conditions (3.1) and $A \in \mathcal{S}\left(\eta, \frac{\pi}{2}\right)$, we can apply results of Ch. 3 of [28] to (4.9).

(i) Theorem 3.2 with Corollary 1.1 and Proposition 1.2 in [28] implies that there exists a family of operators $S:[0, \infty) \rightarrow \mathcal{L}(X)$ (called resolvent of (4.9)) so that a solution $v \in V$ (if it exists) is represented by the formula $v=\frac{d}{d t} S * F$. By assumptions of (i), (4.9) has a solution $v \in V$. Since $F=0$, we have $v=0$. Thus, $u=0$.

(ii) Theorem 3.3 (i) [28] implies that for $F \in C_{0}^{\alpha}([0, T] ; X)$ there exists a solution $v \in V^{\alpha}$ of (4.9). This proves the existence of the solution $u \in$ $C_{0}^{\{k\}, \alpha}([0, T] ; X) \cap C_{0}^{\alpha}\left([0, T] ; X_{A}\right)$ of (4.6). The estimate (4.7) follows from the bounded inverse theorem.

(iii) It is sufficient to prove this assertion in case $F(t) \equiv \xi \in X$, because the problem with given pair of data $(F, \varphi)$ can be splitted into two problems with the data $(F-F(0), 0)$ and $(F(0), \varphi)$, respectively. For the first problem, the assertion (ii) applies. Having proved (iii) for the second one, $u$ is expressed as the sum of solutions of these two problems and satisfies (iii), too.

Thus, let us assume that $F(t) \equiv \xi \in X$. Due to Proposition 1.2 (ii) [28], (4.9) has the solution $v=S(\xi+A \varphi) \in V$. This implies the existence assertion of (iii). Due to the strong continuity of $S(t)[28],\|S(t)\|_{\mathcal{L}(X)} \leq C_{3}, t \in[0, T]$, where $C_{3}$ is a constant. Thus, $\|v\|_{C([0, T], X)} \leq C_{3}\left(\|\xi\|_{X}+\|A \varphi\|_{X}\right)$. Extracting the term $A(M * v)$ from (4.9) and estimating it we obtain $\|A(M * v)\|_{C_{0}([0, T], X)} \leq$ $\left(C_{3}+1\right)\left(\|\xi\|_{X}+\|A \varphi\|_{X}\right)$. Consequently,

$$
\|u\|_{C\{k\}([0, T] ; X) \cap C\left([0, T] ; X_{A}\right)}=\|v\|_{V}+\|\varphi\|_{X_{A}} \leq C_{4}\left(\|\xi\|_{X}+\|\varphi\|_{X_{A}}\right)
$$

with a constant $C_{4}$. This implies (4.8).

\subsection{Statements on direct problem}

In order to apply Lemma 3 to the direct problem (2.6), we must introduce appropriate Banach spaces of $x$-dependent functions and define realizations of the operator $L$ in these spaces so that they belong to $\mathcal{S}\left(\eta, \frac{\pi}{2}\right)$.

Let us introduce the following spaces and operators:

1. $X_{p}=L_{p}(\Omega), 1<p<\infty$, $A_{p}: X_{A_{p}} \rightarrow X_{p}$ with $X_{A_{p}}=\left\{z \in W_{p}^{2}(\Omega):\left.\mathcal{B} z\right|_{\partial \Omega}=0\right\}$ and $A_{p} z=L z, \quad z \in X_{A_{p}}$.

2. $X_{0}=\left\{\begin{array}{l}C_{0}(\bar{\Omega})=\left\{z \in C(\bar{\Omega}):\left.z\right|_{\partial \Omega}=0\right\} \text { in case } \mathcal{B}=I, \\ C(\bar{\Omega}) \text { in case } \mathcal{B}=\omega \cdot \nabla,\end{array}\right.$ $A_{0}: X_{A_{0}} \rightarrow X_{0}$ with $X_{A_{0}}=\left\{z \in \bigcap_{1<p<\infty} W_{p}^{2}(\Omega):\left.\mathcal{B} z\right|_{\partial \Omega}=0, L z \in X_{0}\right\}$ and $A_{0} z=L z, \quad z \in X_{A_{0}}$. 
Corollary 1. Operators $A_{p}, p \in\{0\} \bigcup(1, \infty)$, are sectorial. Thus, Lemma 3 holds in cases $X=X_{p}, A=A_{p}, p \in\{0\} \bigcup(1, \infty)$ and applies to problem (2.6).

Proof. It follows from Theorems 3.1.2, 3.1.3 and Corollary 3.1.24 (ii) in [24].

Lemma 4. Let $K \in L_{1}(0, T) \bigcap C^{1}(0, T), \lim _{t \rightarrow 0^{+}} K(t)=\infty, K>0, K$ be nonincreasing and $\exists t_{K}>0: K$ is strictly decreasing in $\left(0, t_{K}\right)$. Moreover, let $F \in C([0, T] \times \bar{\Omega})$. Assume that $u$ solves the problem

$$
\begin{aligned}
& D_{t}^{\{K\}}(u-\varphi)(t, x)=L u(t, x)+F(t, x), t \in(0, T), x \in \Omega, \\
& u(0, x)=\varphi, x \in \Omega
\end{aligned}
$$

and satisfies the smoothness conditions $u \in C([0, T] \times \bar{\Omega}), u_{x_{j}} \in C((0, T] \times \bar{\Omega})$, $u \in C\left((0, T] ; W_{p}^{2}(\Omega)\right)$ for some $p>n, L_{1} u \in C((0, T] \times \bar{\Omega}), D_{t}^{\{K\}}(u-\varphi) \in$ $C((0, T] \times \bar{\Omega})$. Finally, let

$$
\lim _{\epsilon \rightarrow 0^{+}} \frac{1}{\epsilon} \int_{0}^{\epsilon} K(\tau) d \tau \sup _{0 \leq s \leq \epsilon}|u(t-s, x)-u(t, x)|=0, \quad \forall t \in(0, T], x \in \bar{\Omega} .
$$

If $\varphi \geq 0, F \geq 0$ and $\left.\mathcal{B} u\right|_{\partial \Omega} \geq 0$ then the following assertions are valid.

(i) $u \geq 0$;

(ii) if $u\left(t_{0}, x_{0}\right)=0$ in some point $\left(t_{0}, x_{0}\right) \in(0, T] \times \Omega_{N}$, where

$$
\Omega_{N}= \begin{cases}\Omega & \text { in case } \mathcal{B}=I \\ \bar{\Omega} & \text { in case } \mathcal{B}=\omega \cdot \nabla\end{cases}
$$

then $u\left(t, x_{0}\right)=0$ for any $t \in\left[0, t_{0}\right]$.

This lemma is a slight modification of a positivity principle that was proved in [15] for a semilinear equation in case of a more smooth solution $u \in$ $C\left((0, T] ; C^{2}(\bar{\Omega})\right)$ and strictly decreasing in $(0, T)$ kernel $K$.

To prove Lemma 4 , we need the following auxiliary result. It is proved in Appendix of the paper.

Lemma 5. Let $w \in W_{p}^{2}(\Omega)$ for some $p>n, L_{1} w \in C(\bar{\Omega})$ and $x^{*}=\underset{\bar{\Omega}}{\operatorname{argmin}} w(x)$. In case $x^{*} \in \partial \Omega$ we also assume that $(\omega \cdot \nabla w)\left(x^{*}\right) \geq 0$. Then $\begin{gathered}x \in \bar{\Omega} \\ L_{1} w\left(x^{*}\right)\end{gathered} \geq 0$.

Proof of Lemma 4. Without a restriction of generality we assume that $r \leq 0$. Otherwise it is possible to define $\tilde{u}=e^{-\sigma t} u$ as in [15] and to consider the corresponding problem for $\tilde{u}$. Such a problem also satisfies the assumptions of Lemma 4 and has the coefficient $\tilde{r}=r-\sigma \int_{0}^{T} e^{-\sigma s} K(s) d s$ in place of $r$. Since $\lim _{t \rightarrow 0^{+}} K(t)=\infty$, for sufficiently large $\sigma, \tilde{r} \leq 0$.

Let us suppose that (i) does not hold. Then there exists $\left(t_{1}, x_{1}\right) \in(0, T] \times \bar{\Omega}$ such that $u\left(t_{1}, x_{1}\right)<0$ and $\left(t_{1}, x_{1}\right)=\underset{x \in \bar{\Omega}, t \in[0, T]}{\operatorname{argmin}} u(t, x)$. It was shown in $[15]$ (formula (37)) that the assumptions $D_{t}^{\{K\}}(u-\varphi) \in C((0, T] \times \bar{\Omega}),(4.10)$, 
$K>0$ and $K$ - nonincreasing together with the relations $u\left(t, x_{1}\right) \geq u\left(t_{1}, x_{1}\right)$ and $u\left(t_{1}, x_{1}\right)<0$ imply $D_{t}^{\{K\}}(u-\varphi)\left(t_{1}, x_{1}\right)<0$. On the other hand, Lemma 5 applies to the function $w=u\left(t_{1}, \cdot\right)$ at $x^{*}=x_{1}$. We obtain $L_{1} u\left(t_{1}, x_{1}\right) \geq 0$. Also $r\left(x_{1}\right) u\left(t_{1}, x_{1}\right) \geq 0$ and $F \geq 0$. Thus, the left-hand side of the equation $D_{t}^{\{K\}}(u-\varphi)\left(t_{1}, x_{1}\right)=[L u+F]\left(t_{1}, x_{1}\right)$ is negative, but the right-hand side is nonnegative. We have reached a contradiction. The assertion (i) is valid.

Let us prove (ii). Let $u\left(t_{0}, x_{0}\right)=0$ at $\left(t_{0}, x_{0}\right) \in(0, T] \times \Omega_{N}$. Define $\hat{t}_{0}=\inf \left\{t: t \leq t_{0}, u\left(\tau, x_{0}\right)=0\right.$ for $\left.\tau \in\left[t, t_{0}\right]\right\}$. If (ii) is not valid, then $\hat{t}_{0}>0$ and $u\left(t, x_{0}\right) \geq \delta, t \in\left(t_{2}, t_{3}\right)$ for some $\delta>0$ and $\left(t_{2}, t_{3}\right) \subset\left(0, \hat{t}_{0}\right)$ such that $\hat{t}_{0}-t_{2}<t_{K}$. Then, similarly to the proof in [15] p.138, from the assumptions $D_{t}^{\{K\}}(u-\varphi) \in C((0, T] \times \bar{\Omega}),(4.10), K>0, K-$ nonincreasing and relations $u \geq 0, u\left(t, x_{0}\right) \geq \delta>0, t \in\left(t_{2}, t_{3}\right)$, we derive

$$
D_{t}^{\{K\}}(u-\varphi)\left(\hat{t}_{0}, x_{0}\right) \leq \delta\left(K\left(\hat{t}_{0}-t_{2}\right)-K\left(\hat{t}_{0}-t_{3}\right)\right)
$$

Since $0<\hat{t}_{0}-t_{3}<\hat{t}_{0}-t_{2}<t_{K}$ and $K$ is strictly decreasing in $\left(0, t_{K}\right),(4.11)$ implies $D_{t}^{\{K\}}(u-\varphi)\left(\hat{t}_{0}, x_{0}\right)<0$. On the other hand, from $u\left(\hat{t}_{0}, x_{0}\right)=0$ and $u(t, x) \geq 0,(t, x) \in(0, T] \times \Omega$, we conclude that $\left(\hat{t}_{0}, x_{0}\right)=\underset{x \in \bar{\Omega}}{\operatorname{argmin}} u\left(\hat{t}_{0}, x\right)$. By Lemma $5, L_{1} u\left(\hat{t}_{0}, x_{0}\right) \geq 0$. Moreover, $(r u)\left(\hat{t}_{0}, x_{0}\right)=0$ and $F \geq 0$. Lefthand side of the equation $D_{t}^{\{K\}}(u-\varphi)\left(\hat{t}_{0}, x_{0}\right)=[L u+F]\left(\hat{t}_{0}, x_{0}\right)$ is negative, but right-hand side is nonnegative. Again, we have reached the contradiction. Thus, (ii) holds.

At this point we present somewhat more concrete assumptions on the input data of the direct problem (2.6) that imply the assumptions of Lemma 4 and Lemma 3.

Corollary 2. Let $F \geq 0, \varphi=0$ and one of the assumptions (a1)-(a3) hold:

(a1) $F \in C^{\{M\}, \alpha}\left([0, T] ; X_{0}\right)$ for some $0<\alpha<1$ and $F(0, \cdot)=0$;

(a2) $F \in C_{0}^{\alpha}\left([0, T] ; X_{0}\right)$ and $M(t) \geq c t^{\gamma-1}, t \in(0, T)$ for some $c \in \mathbb{R}_{+}$, $0<\gamma<\alpha<1$

(a3) $F \in C_{0}^{\alpha-\beta}\left([0, T] ; X_{0}\right)$ and $c_{1} t^{\gamma-1} \leq M(t) \leq c_{2} t^{\beta-1},\left|M^{\prime}(t)\right| \leq c_{3} t^{\beta-2}$, $t \in(0, T)$, for some $c_{1}, c_{2}, c_{3} \in \mathbb{R}_{+}, 0<\beta \leq \gamma<\alpha<1$.

Then assertions Lemma 4 are satisfied by solution of the problem (2.6).

Proof. Defining $X=X_{0}$, Lemma 3 with Corollary 1 implies that the solution of (2.6) exists and satisfies the smoothness conditions of Lemma 4. It remains to show that (4.10) holds.

The case (a1). The relations $F \in C^{\{M\}, \alpha}\left([0, T] ; X_{0}\right), F(0, \cdot)=0$ mean that $F=k * \hat{F}$, where $\hat{F} \in C^{\alpha}\left([0, T] ; X_{0}\right)$. Thus, it follows from Lemma 3 that the function $\hat{u}$ that solves $(2.6)$ with $F, \varphi$ replaced by $\hat{F}, \hat{\varphi}=0$ belongs to the space $C^{\{k\}}\left([0, T] ; X_{0}\right)$. Next, after convolving equation for $\hat{u}$ with $k$ it is easy to see that $u=k * \hat{u}$ solves $(2.6)$ with $F=k * \hat{F}$. Therefore, $u \in k * C^{\{k\}}\left([0, T] ; X_{0}\right)$, that is $u=k * M * v=1 * v, v \in C\left([0, T] ; X_{0}\right)$. This allows us to conclude that 
$u \in C^{1}\left([0, T] ; X_{0}\right)$. Hence,

$$
\begin{aligned}
\lim _{\epsilon \rightarrow 0^{+}} & \frac{1}{\epsilon} \int_{0}^{\epsilon} k(\tau) d \tau \sup _{0 \leq s \leq \epsilon}|u(t-s, x)-u(t, x)|=\lim _{\epsilon \rightarrow 0^{+}} \frac{1}{\epsilon} \int_{0}^{\epsilon} k(\tau) d \tau \cdot O(\epsilon) \\
& =0, \quad \forall t \in(0, T], x \in \bar{\Omega} .
\end{aligned}
$$

The case (a2). Again, by Lemma 3 (ii), $u \in C_{0}^{\{k\}, \alpha}\left([0, T] ; X_{0}\right)$ and by (4.5), $u \in C_{0}^{\alpha}\left([0, T] ; X_{0}\right)$. The relation (4.10) follows from the estimate

$$
\begin{aligned}
& \lim _{\epsilon \rightarrow 0^{+}} \frac{1}{\epsilon} \int_{0}^{\epsilon} k(\tau) d \tau \sup _{0 \leq s \leq \epsilon}|u(t-s, x)-u(t, x)|=\lim _{\epsilon \rightarrow 0^{+}} \frac{1}{\epsilon} \int_{0}^{\epsilon} k(\tau) d \tau \cdot O\left(\epsilon^{\alpha}\right) \\
& \leq \lim _{\epsilon \rightarrow 0^{+}} \frac{O\left(\epsilon^{\alpha}\right)}{\epsilon M(\epsilon)} \int_{0}^{\epsilon} M(\epsilon-\tau) k(\tau) d \tau=\lim _{\epsilon \rightarrow 0^{+}} O\left(\epsilon^{\alpha-\gamma}\right)=0 \quad \forall t \in(0, T], x \in \bar{\Omega} .
\end{aligned}
$$

The case (a3). According to Lemma 3 (ii), $F \in C_{0}^{\alpha-\beta}\left([0, T] ; X_{0}\right)$ implies that $u \in C_{0}^{\{k\}, \alpha-\beta}\left([0, T] ; X_{0}\right)=M * C_{0}^{\alpha-\beta}\left([0, T] ; X_{0}\right)$. By Lemma 2 it holds $u \in C_{0}^{\alpha}\left([0, T] ; X_{0}\right)$. This enables us finish the proof as in case (a2).

\section{Results on IP1}

We will study IP1 in context of Hölder spaces with respect to $t$. For the sake of generality, we will assume different orders of spaces related to $g$ and $h$ : for $g$ we use $\alpha_{1}$ and for $h$ we use $\alpha_{2}$.

Theorem 1. Let one of the following assumptions be valid:

(A1) $g \in C_{0}^{1+\alpha_{1}}([0, T] ; C(\bar{\Omega}))$ for some $0<\alpha_{1}<1$;

(A2) $g \in C_{0}^{\{k\}, \alpha_{1}}([0, T] ; C(\bar{\Omega}))$ and $M(t) \geq c t^{\gamma-1}, t \in(0, T)$ for some $c \in \mathbb{R}_{+}$, $0<\gamma<\alpha_{1}<1$

(A3) $g \in C_{0}^{\{k\}, \alpha_{1}-\beta}([0, T] ; C(\bar{\Omega}))$ and $c_{1} t^{\gamma-1} \leq M(t) \leq c_{2} t^{\beta-1},\left|M^{\prime}(t)\right| \leq$ $c_{3} t^{\beta-2}, t \in(0, T)$, for some $c_{1}, c_{2}, c_{3} \in \mathbb{R}_{+}, 0<\bar{\beta} \leq \gamma<\alpha_{1}<1$.

Additionally, we assume that $g \geq 0, g_{1}:=D_{t}^{\{k\}} g-R g \geq 0$ where $R:=\max _{x \in \bar{\Omega}} r(x)$ and

$$
\text { a.e. } x \in \Omega \quad \exists t_{x} \in(0, T]: g\left(t_{x}, x\right)>0 \text {. }
$$

In case $\mathcal{B}=I$ we also assume that $\forall x \in \partial \Omega$, either $g(T, x)>0$ or $g(\cdot, x)=0$.

Finally, let $(f, u) \in C(\bar{\Omega}) \times\left(C_{0}^{\{k\}}([0, T] ; C(\bar{\Omega})) \bigcap C_{0}\left([0, T] ; W_{p}^{2}(\Omega)\right)\right)$ for some $p>1$ solve IP1 for $\varphi=0, \psi=0, h=0$. Then $(f, u)=(0,0)$.

Proof. We start the proof by showing that in case $\mathcal{B}=I$, for any $x \in \partial \Omega$ such that $g(T, x)>0$, the equality $f(x)=0$ is valid. To show this, we consider the equality

$$
D_{t}^{\{k\}} u(T, x)=f(x) g(T, x), \quad x \in \bar{\Omega},
$$

that follows from equation (2.6) in view of $\psi=0$. If $x \in \partial \Omega$ and $\mathcal{B}=I$ then the left-hand side of this equality equals zero. Thus, $f(x) g(T, x)=0$ and provided $g(T, x)>0$ we obtain $f(x)=0$. 
Let us introduce the functions $f^{+}=\frac{|f|-f}{2}$ and $f^{-}=\frac{|f|+f}{2}$. Due to the definition, $f^{ \pm} \in C(\bar{\Omega})$ and $f^{ \pm} \geq 0$. Moreover,

in case $\mathcal{B}=I$, for any $x \in \partial \Omega$ such that $g(T, x)>0$, it holds $f^{ \pm}(x)=0$. (5.2)

Firstly, we consider the problems

$$
\begin{aligned}
& D_{t}^{\{k\}} u^{ \pm}(t, x)=L u^{ \pm}(t, x)+g(t, x) f^{ \pm}(x), \quad x \in \Omega, t \in(0, T), \\
& u^{ \pm}(0, x)=0, \quad x \in \Omega, \quad \mathcal{B} u^{ \pm}(t, x)=0, \quad x \in \partial \Omega, t \in(0, T) .
\end{aligned}
$$

By assumptions of the theorem and $(5.2), g(t, \cdot) f^{ \pm} \in X_{0}, t \in[0, T]$. Therefore, in cases (A1) and (A2) due to (4.5) we have $g f^{ \pm} \in C_{0}^{\{M\}, \alpha_{1}}\left([0, T] ; X_{0}\right)$ and $g f^{ \pm} \in C_{0}^{\alpha_{1}}\left([0, T] ; X_{0}\right)$, respectively. Similarly, in case (A3) due to (4.5) and Lemma 2 we obtain $g f^{ \pm} \in C_{0}^{\alpha_{1}}\left([0, T] ; X_{0}\right)$. Moreover, $g f^{ \pm} \geq 0$. The assumptions of Corollary 2 are satisfied for the functions $F=g f^{ \pm}$. Hence, the solutions $u^{ \pm}$of (5.3) satisfy the assertions of Lemma 4 .

Secondly, let us consider the problems

$$
\begin{aligned}
& D_{t}^{\{k\}} v^{ \pm}(t, x)=L v^{ \pm}(t, x)+g_{1}(t, x) f^{ \pm}(x), \quad x \in \Omega, t \in(0, T), \\
& v^{ \pm}(0, x)=0, \quad x \in \Omega, \quad \mathcal{B} v^{ \pm}(t, x)=0, \quad x \in \partial \Omega, t \in(0, T) .
\end{aligned}
$$

In case $(\mathrm{A} 1)$ we have $g^{\prime} \in C_{0}^{\alpha_{1}}([0, T] ; C(\bar{\Omega}))$. Thus, $g_{1}=D_{t}^{\{k\}} g-R g=$ $k * g^{\prime}-R g \in C_{0}^{\{M\}, \alpha_{1}}([0, T] ; C(\bar{\Omega}))$. From $g(t, \cdot) f^{ \pm} \in X_{0}, t \in[0, T]$ we immediately get $g_{1}(t, \cdot) f^{ \pm} \in X_{0}, t \in[0, T]$. Therefore, $g_{1} f^{ \pm} \in C_{0}^{\{M\}, \alpha_{1}}\left([0, T] ; X_{0}\right)$.

Using similar reasoning, we deduce $g_{1} f^{ \pm} \in C_{0}^{\alpha_{1}}\left([0, T] ; X_{0}\right)$ and $g_{1} f^{ \pm} \in$ $C_{0}^{\alpha_{1}-\beta}\left([0, T] ; X_{0}\right)$ in cases (A2) and (A3), respectively. Moreover, $g_{1} f^{ \pm} \geq 0$. Again, the assumptions of Corollary 2 are satisfied for $F=g_{1} f^{ \pm}$. The solutions $v^{ \pm}$of (5.4) satisfy the assertions of Lemma 4 .

Let us point out that the problem for $M * v^{ \pm}$is equivalent to the problem for $u^{ \pm}-R M * u^{ \pm}$. Thus,

$$
v^{ \pm}=D_{t}^{\{k\}} u^{ \pm}-R u^{ \pm}
$$

Moreover, since $f=f^{+}-f^{-}$, we have $u=u^{+}-u^{-}$. Thus, $\psi=u(T, \cdot)=$ 0 implies that $u^{+}(T, \cdot)=u^{-}(T, \cdot)$. Let us denote $x^{*}=\operatorname{argmax} u^{+}(T, x)=$ $\operatorname{argmax} u^{-}(T, x)$. By definition, either $f^{+}\left(x^{*}\right)=0$ or $f^{-}\left(x^{*}\right)=0$. Let us $x \in \bar{\Omega}$

assume that $f^{+}\left(x^{*}\right)=0$ (the situation when $f^{-}\left(x^{*}\right)=0$ can be considered in a similar manner).

Let us suppose that either $x^{*} \in \Omega$ or $\mathcal{B}=\omega \cdot \nabla$ (the case $x^{*} \in \partial \Omega$ and $\mathcal{B}=I$ will be considered later separately). Then we can apply Lemma 5 to the function $w=-u^{+}(T, \cdot)$. We get $L_{1} u^{+}\left(T, x^{*}\right) \leq 0$. Thus, from (5.3), (5.5) and $u^{+} \geq 0, r \leq R$ it follows:

$$
v^{+}\left(T, x^{*}\right)=L_{1} u^{+}\left(T, x^{*}\right)+\left(r\left(x^{+}\right)-R\right) u^{+}\left(T, x^{*}\right) \leq 0 .
$$

Due to Lemma 4 (i),

$$
v^{+}(t, x) \geq 0,(t, x) \in(0, T) \times \Omega .
$$


Hence, (5.6) and (5.7) imply $v^{+}\left(T, x^{*}\right)=0$. Thus, by Lemma 4 (ii), $v^{+}\left(t, x^{*}\right)=$ $0, t \in[0, T]$. By formula (5.5) it means $D_{t}^{\{k\}} u^{+}\left(t, x^{*}\right)-R u^{+}\left(t, x^{*}\right)=0, t \in$ $[0, T]$. Applying $M *$ to to this equality, we obtain the following homogeneous Volterra equation of the second kind:

$$
u^{+}\left(t, x^{*}\right)-R M * u^{+}\left(t, x^{*}\right)=0, \quad t \in[0, T] .
$$

It has only the trivial solution $u^{+}\left(t, x^{*}\right)=0, t \in[0, T]$. Hence, $u^{+}\left(T, x^{*}\right)=0$.

Since $x^{*}$ is a maximum point of $u^{+}(T, x)$ and $u^{+}(T, x) \geq 0$, we also get

$$
u^{+}(T, x)=0, \quad x \in \Omega \text {. }
$$

Now we consider the case $x^{*} \in \partial \Omega, \mathcal{B}=I$, too. Then by $\left.\mathcal{B} u^{+}\right|_{\partial \Omega}=0$, immediately $u^{+}\left(T, x^{*}\right)=0$ and again we have (5.8).

Since $u=u^{+}-u^{-}$and $\psi=u(T, \cdot)=0$ holds, from (5.8) we get $u^{ \pm}(T, x)=0$, $x \in \Omega$. Lemma 4 (ii) implies $u^{ \pm}(t, x)=0,(t, x) \in[0, T] \times \Omega$. Therefore, $u(t, x)=0,(t, x) \in[0, T] \times \Omega$. From the differential equation for $u$ we obtain $f(x) g(t, x)=0,(t, x) \in[0, T] \times \Omega$. Finally, (5.1) yields $f=0$.

Next we provide simple sufficient conditions that imply the assumption $D_{t}^{\{k\}} g-R g \geq 0$ in Theorem 1. For this we need the following lemma.

Lemma 6. Let $w \in C^{\{k\}}([0, T] ; \mathbb{R})$ be nonnegative and nonincreasing. Then $D_{t}^{\{k\}} w \geq k(T) w$.

Proof. The assertion follows from the estimate

$$
\begin{aligned}
D_{t}^{\{k\}} w(t)=\lim _{\delta \rightarrow 0^{+}} \frac{1}{\delta}\left[\int_{t}^{t+\delta} k(\tau) w(t+\delta-\tau) d \tau+\int_{0}^{t} k(\tau)(w(t+\delta-\tau)\right. \\
-w(t-\tau)) d \tau] \geq \lim _{\delta \rightarrow 0^{+}} k(T+\delta) \frac{1}{\delta}\left[\int_{t}^{t+\delta} w(t+\delta-\tau) d \tau+\int_{0}^{t}(w(t+\delta-\tau)\right. \\
-w(t-\tau)) d \tau]=k(T) w(t), \quad 0<t<T .
\end{aligned}
$$

Due to that Lemma $6, D_{t}^{\{k\}} g-R g \geq 0$ holds provided along with other assumptions on $g$ in Theorem $1, g$ is nondecreasing in $t$ and $k(T) \geq R$ in case $R>0$.

Theorem 2. Let $g, M$ satisfy the assumptions of Theorem 1 and the inequality $g(T, x)>0, x \in \bar{\Omega}$, hold. If $\varphi, \psi \in X_{A_{p}}$ and $h \in C^{\alpha_{2}}\left([0, T] ; X_{p}\right)$, where $p \in\{0\} \bigcup(1, \infty), 0<\alpha_{2}<1$, then IP1 has a unique solution $(f, u) \in X_{p} \times$ $C^{\{k\}}\left([0, T] ; X_{p}\right) \cap C\left([0, T] ; X_{A_{p}}\right)$ and the following estimate holds:

$$
\begin{aligned}
& \|f\|_{X_{p}}+\|u\|_{C\{k\}\left([0, T] ; X_{p}\right)} \cap C\left([0, T] ; X_{A_{p}}\right) \\
& \quad \leq C_{5}\left(\|\varphi\|_{X_{A_{p}}}+\|\psi\|_{X_{A_{p}}}+\|h\|_{\left.C^{\alpha_{2}\left([0, T] ; X_{p}\right)}\right)}\right)
\end{aligned}
$$


If additionally $\varphi=h(0, \cdot)=0$, then $u \in C_{0}^{\{k\}, \alpha}\left([0, T] ; X_{p}\right) \bigcap C_{0}^{\alpha}\left([0, T] ; X_{A_{p}}\right)$ where $\alpha=\left\{\begin{array}{ll}\alpha_{2}, & \text { in case (A1), } \\ \min \left\{\alpha_{1}, \alpha_{2}\right\}, & \text { in cases (A2), (A3) }\end{array}\right.$ and the estimate

$$
\|f\|_{X_{p}}+\|u\|_{C_{0}^{\{k\}, \alpha}\left([0, T] ; X_{p}\right) \cap C_{0}^{\alpha}\left([0, T] ; X_{A_{p}}\right)} \leq C_{6}\left(\|\psi\|_{X_{A_{p}}}+\|h\|_{C_{0}^{\alpha_{2}}\left([0, T] ; X_{p}\right)}\right)(5
$$

is valid. The constants $C_{5}$ and $C_{6}$ depend on the parameters $M, L, g, p, \alpha_{2}$.

Proof. Firstly, we are going to replace the overdetermination condition (2.9) by a fixed-point equation with respect to $f$.

Suppose that $(f, u) \in X_{p} \times C^{\{k\}}\left([0, T] ; X_{p}\right) \bigcap C\left([0, T] ; X_{A_{p}}\right)$ solves IP1. Then, since (2.9) holds, the equation (2.6) at $t=T$ with $F=f g+h$ yields

$$
f(x)=\frac{\left(D_{t}^{\{k\}}(u-\varphi)-\eta u\right)(T, x)-\left(A_{p}-\eta\right) \psi(x)-h(T, x)}{g(T, x)}
$$

where $\eta$ is chosen so that $0 \in \rho\left(A_{p}-\eta I\right)$.

Let us split $u$ into the sum of two functions: $u=u_{1}+u_{2}$, such that

$$
\begin{aligned}
& D_{t}^{\{k\}} u_{1}=A_{p} u_{1}+f g, \quad u_{1}(0, \cdot)=0, \\
& D_{t}^{\{k\}}\left(u_{2}-\varphi\right)=A_{p} u_{2}+h, \quad u_{2}(0, \cdot)=\varphi .
\end{aligned}
$$

In the context of IP1, $u_{2}$ is a known function. According to Lemma 3 , the solution to $(5.12)$ belongs to $u_{2} \in C^{\{k\}}\left([0, T] ; X_{p}\right)$. Thus, $v_{2}:=D_{t}^{\{k\}}\left(u_{2}-\varphi\right)-$ $\eta u_{2} \in C\left([0, T] ; X_{p}\right)$. Next we formulate the following problem:

$$
D_{t}^{\{k\}} v_{1}=A_{p} v_{1}+f\left(D_{t}^{\{k\}} g-\eta g\right), \quad v_{1}(0, \cdot)=0 .
$$

Due to the assumptions (A1)-(A3) and (4.5), it holds $D_{t}^{\{k\}} g \in C_{0}^{\hat{\alpha}}([0, T] ; C(\bar{\Omega}))$ where

$$
\hat{\alpha}= \begin{cases}\alpha_{1}, & \text { in cases (A1), (A2), } \\ \alpha_{1}-\beta, & \text { in case (A3). }\end{cases}
$$

Thus, $f\left(D_{t}^{\{k\}} g-\eta g\right) \in C_{0}^{\hat{\alpha}}\left([0, T] ; X_{p}\right)$. According to Lemma 3, (5.13) has a solution $v_{1}$ in $C_{0}^{\{k\}, \hat{\alpha}}\left([0, T] ; X_{p}\right) \bigcap C_{0}^{\hat{\alpha}}\left([0, T] ; X_{A_{p}}\right)$. It is easy to check that $v_{1}=D_{t}^{\{k\}} u_{1}-\eta u_{1}$.

The notations introduced allow us to rewrite (5.11) in the form

$$
f=\mathcal{F} f+\mathcal{G},
$$

where

$$
\begin{aligned}
& \mathcal{G}(x)=\frac{v_{2}(T, x)-\left(A_{p}-\eta\right) \psi(x)-h(T, x)}{g(T, x)}, \quad x \in \Omega, \\
& (\mathcal{F} f)(x)=v_{1}[f](T, x) / g(T, x)
\end{aligned}
$$


and $v_{1}[\cdot]$ stands for the operator that assigns to $f$ the solution $v_{1}$ of (5.13). Thus, (2.6), (2.9), (2.10) imply (5.15). On the other hand, taking into account all the substitutions performed, we can move back from (5.15) to (5.11). Together with (2.6) at $t=T$ and (2.10) it implies $\left(A_{p}-\eta\right) u(T, x)=\left(A_{p}-\eta\right) \psi(x)$. Since $\left(A_{p}-\eta\right)$ is injective, it yields $(2.9)$. Consequently, IP1 is in the space $X_{p} \times C^{\{k\}}\left([0, T] ; X_{p}\right) \bigcap C\left([0, T] ; X_{A_{p}}\right)$ equivalent to the problem of finding the pair of functions $(f, u)$ that solves $(2.6),(2.10),(5.15)$.

We point out that (5.15) is an independent equation for the first component $f$ of the solution of IP1. Let us analyse properties of the operator $\mathcal{F}$ involved in this equation. By Lemma $3, v_{1}[\cdot] \in \mathcal{L}\left(X_{p} ; C_{0}^{\hat{\alpha}}\left([0, T] ; X_{A_{p}}\right)\right)$. Thus, $v_{1}[\cdot](T, \cdot) \in$ $\mathcal{L}\left(X_{p}, X_{A_{p}}\right)$.

Furthermore, $X_{A_{p}} \hookrightarrow \hookrightarrow X_{p}$. In case $p \in(1, \infty)$ it is a direct consequence of $W_{p}^{2}(\Omega) \hookrightarrow \hookrightarrow L_{p}(\Omega)$. In case $p=0$ it follows from the continuous embedding of $X_{A_{0}}$ in $C_{\mathcal{B}}^{1}(\bar{\Omega}):=X_{0} \bigcap C^{1}(\bar{\Omega})$ (see Theorems 3.1.19, 3.1.22 in [24]) and $C_{\mathcal{B}}^{1}(\bar{\Omega}) \hookrightarrow \hookrightarrow X_{0}$.

Therefore, $v_{1}[\cdot](T, \cdot): X_{p} \rightarrow X_{p}$ is compact. Since $\frac{1}{g(T, \cdot)} \in C(\bar{\Omega})$ due to the assumptions of this theorem, $\mathcal{F}: X_{p} \rightarrow X_{p}$ is also compact.

Next, let us show that $1 \notin \sigma(\mathcal{F})$. Firstly, let us consider the case $p=0$. Suppose that $1 \in \sigma(\mathcal{F})$. Then the equation $f=\mathcal{F} f$ has a solution $f \in X_{0}$, $f \neq 0$. This means that the problem (2.6), (2.10), (5.15) with homogeneous data $\varphi=0, \psi=0, h=0$ has the nontrivial solution $\left(f, u_{1}\right)$ in the space $X_{0} \times C_{0}^{\{k\}}\left([0, T] ; X_{0}\right) \cap C_{0}\left([0, T] ; X_{A_{0}}\right)$. But due to the Theorem 1 , IP1 with a homogeneous data has only the trivial solution in such a space. We came to a contradiction. Consequently, $1 \notin \sigma(\mathcal{F})$.

Secondly, let us consider the case $p \in(1, \infty)$. We again suppose that $1 \in \sigma(\mathcal{F})$, hence the equation $f=\mathcal{F} f$ has a nontrivial solution $f \in X_{p}$. The idea is to show that this solution actually belongs to $X_{0}$. Then we can apply the arguments from the previous case to show that $1 \in \sigma(\mathcal{F})$ leads to a contradiction.

If $p>\frac{n}{2}$, then $v_{1}[f](T, \cdot) \in X_{A_{p}} \hookrightarrow X_{0}$. Thus, $f=\mathcal{F} f=\frac{1}{g(T, x)} v_{1}[f](T, \cdot) \in$ $X_{0}$. If $p \leq \frac{n}{2}$, then according to embedding theorems, $X_{A_{p}} \hookrightarrow X_{p_{1}}=L_{p_{1}}(\Omega)$, where $p_{1}=\frac{n p}{n-2 p}>p$. Therefore, $v_{1}[f](T, \cdot) \in X_{p_{1}}$ and $f=\mathcal{F} f=$ $\frac{1}{g(T, x)} v_{1}[f](T, \cdot) \in X_{p_{1}}$. After a finite number of iterations we obtain $f \in X_{p_{i}}$, where $p_{i}=\frac{n p}{n-2 i p}>\frac{n}{2}$ (works for $i>\frac{n}{2 p}-1$ ). Next iteration gives $f \in X_{0}$.

We have shown that the first case of Fredholm alternative is satisfied for the equation (5.15). Consequently, the solution to (5.15) exists and is unique for any $\mathcal{G} \in X_{p}$ and $(I-\mathcal{F})^{-1} \in \mathcal{L}\left(X_{p}\right)$.

Since $F=f g+h$ is Hölder-continuous with values in $X_{p}$, Lemma 3 implies that the problem $(2.6),(2.10)$ has unique solution $u \in C^{\{k\}}\left([0, T] ; X_{p}\right) \bigcap$ $C\left([0, T] ; X_{A_{p}}\right)$. This completes the proof of the existence and uniqueness assertion of the theorem.

In the rest of the proof, $\widehat{C}$ stands for a generic constant depending on the parameters $M, L, g, p, \alpha_{2}$. Let us deduce the stability estimate (5.9). We obtain

$$
\|f\|_{X_{p}} \leq\left\|(I-\mathcal{F})^{-1}\right\|_{\mathcal{L}\left(X_{p}\right)}\|\mathcal{G}\|_{X_{p}} \leq \widehat{C}\left(\|h(T, \cdot)\|_{X_{p}}+|\eta|\|\psi\|_{X_{p}}+\|\psi\|_{X_{A_{p}}}\right.
$$




$$
\begin{aligned}
& \left.+\left\|D_{t}^{\{k\}}\left(u_{2}-\varphi\right)-\eta u_{2}\right\|_{C\left([0, T] ; X_{p}\right)}\right) \leq \widehat{C}\left(\|h\|_{C^{\alpha_{2}\left([0, T] ; X_{p}\right)}}+\|\psi\|_{X_{A_{p}}}\right. \\
& \left.\quad+\|\varphi\|_{X_{A_{p}}}\right) .
\end{aligned}
$$

Further, we note that $g \in C_{0}^{\gamma}([0, T] ; C(\bar{\Omega}))$ for any $\gamma \in(0,1)$ in case (A1) and for $\gamma=\alpha_{1}$ in cases (A2), (A3). Using Lemma 3 we have

$$
\begin{gathered}
\|u\|_{C^{\{k\}}\left([0, T] ; X_{p}\right) \cap C\left([0, T] ; X_{A_{p}}\right)}=\left\|u_{1}+u_{2}\right\|_{C^{\{k\}}\left([0, T] ; X_{p}\right) \cap C\left([0, T] ; X_{A_{p}}\right)} \\
\leq \widehat{C}\left(\|f\|_{X_{p}}\|g\|_{C_{0}^{\gamma}([0, T] ; C(\bar{\Omega}))}+\|h\|_{\left.C^{\alpha_{2}\left([0, T] ; X_{p}\right)}+\|\varphi\|_{X_{A_{p}}}\right) .}\right.
\end{gathered}
$$

Together with the estimate of $f$ (5.18) it implies (5.9).

In case $\varphi=h(0, \cdot)=0$, the solution of (2.6), (2.10) belongs to the space $C_{0}^{\{k\}, \alpha}\left([0, T] ; X_{p}\right) \bigcap C_{0}^{\alpha}\left([0, T] ; X_{A_{p}}\right)$ and can be estimated as

$\|u\|_{C_{0}^{\{k\}, \alpha}\left([0, T] ; X_{p}\right) \cap C_{0}^{\alpha}\left([0, T] ; X_{A_{p}}\right)} \leq \widehat{C}\left(\|f\|_{X_{p}}\|g\|_{C_{0}^{\gamma}([0, T] ; C(\bar{\Omega}))}+\|h\|_{C_{0}^{\alpha_{2}}\left([0, T] ; X_{p}\right)}\right)$.

This with (5.18) implies (5.10).

We point out that in case $p=0$ and $\mathcal{B}=I$, the assumptions of Theorem 2 allow to recover $f \in X_{0}=C_{0}(\bar{\Omega})$ only. In order to fix that in the following theorem we provide some additional conditions that are sufficient to restore $f \in C(\bar{\Omega})$ in case $\mathcal{B}=I$.

Theorem 3. Let $g, M$ satisfy the assumptions of Theorem 2. If $\varphi, \psi, L \varphi \in$ $X_{A_{p}}$ for some $p>\frac{n}{2}, L \psi \in C(\bar{\Omega}), h \in C^{\{k\}, \alpha_{2}}\left([0, T] ; X_{p}\right) \cap C([0, T] ; C(\bar{\Omega}))$, where $0<\alpha_{2}<1$ and $h(0, \cdot) \in X_{A_{p}}$ then $\operatorname{IP1}$ has a unique solution $(f, u) \in$ $C(\bar{\Omega}) \times C^{\{k\}}\left([0, T] ; X_{A_{p}}\right)$. Moreover, Lu $\in C([0, T] ; C(\bar{\Omega}))$ and the estimate

$$
\begin{aligned}
& \|f\|_{C(\bar{\Omega})}+\|u\|_{C\{k\}\left([0, T] ; X_{A_{p}}\right)}+\|L u\|_{C([0, T] ; C(\bar{\Omega}))} \leq C_{7}\left(\|\varphi\|_{X_{p}}+\|L \varphi\|_{X_{A_{p}}}\right. \\
& \left.+\|\psi\|_{X_{p}}+\|L \psi\|_{C(\bar{\Omega})}+\|h\|_{C\{k\}, \alpha_{2}\left([0, T] ; X_{p}\right) \cap C([0, T] ; C(\bar{\Omega}))}+\|h(0, \cdot)\|_{X_{A_{p}}}\right)
\end{aligned}
$$

holds. If additionally $\varphi=h(0, \cdot)=D_{t}^{\{k\}} h(0, \cdot)=0$, then $u \in C_{0}^{\{k\}, \alpha^{\prime}}\left([0, T] ; X_{A_{p}}\right)$ and the estimate

$$
\begin{aligned}
& \|f\|_{C(\bar{\Omega})}+\|u\|_{C_{0}^{\{k\}, \alpha^{\prime}}\left([0, T] ; X_{A_{p}}\right)}+\|L u\|_{C_{0}([0, T] ; C(\bar{\Omega}))} \\
& \leq C_{8}\left(\|\psi\|_{X_{p}}+\|L \psi\|_{C(\bar{\Omega})}+\|h\|_{C_{0}^{\{k\}, \alpha_{2}}\left([0, T] ; X_{p}\right) \cap C_{0}([0, T] ; C(\bar{\Omega}))}\right)
\end{aligned}
$$

is valid where $\alpha^{\prime}=\min \left\{\hat{\alpha} ; \alpha_{2}\right\}$ and $\hat{\alpha}$ is given by (5.14). The constants $C_{7}$ and $C_{8}$ depend on $M, L, g, p, \alpha_{2}$.

Proof. Throughout the proof, $\widehat{C}$ denotes a generic constant depending on $M, L, g, p, \alpha_{2}$ and RHS stands for the expression in brackets at the righthand side of (5.19). By Theorem 2, IP1 has a unique solution $(f, u) \in X_{p} \times$ $C^{\{k\}}\left([0, T] ; X_{p}\right) \bigcap C\left([0, T] ; X_{A_{p}}\right)$. Let us consider the problem

$$
D_{t}^{\{k\}}\left(w_{2}-w_{2}(0, \cdot)\right)=A_{p} w_{2}+D_{t}^{\{k\}}(h-h(0, \cdot)), w_{2}(0, \cdot)=L \varphi+h(0, \cdot) .
$$


Under the assumptions of this theorem, Lemma 3 implies that (5.21) has a unique solution $w_{2} \in C^{\{k\}}\left([0, T] ; X_{p}\right) \cap C\left([0, T] ; X_{A_{p}}\right)$. Moreover, due to (4.7) and (4.8), $\left\|w_{2}\right\|_{C\left([0, T] ; X_{A_{p}}\right)} \leq \widehat{C}\left(\|h\|_{C\{k\}, \alpha_{2}\left([0, T] ; X_{p}\right)}+\|h(0, \cdot)\|_{X_{A_{p}}}+\|L \varphi\|_{X_{A_{p}}}\right)$. It is easy to check that $w_{2}=D_{t}^{\{k\}} *\left(u_{2}-\varphi\right)$ and $u_{2}=M * w_{2}+\varphi$ where $u_{2}$ solves (5.12). Therefore, we have $u_{2} \in C^{\{k\}}\left([0, T] ; X_{A_{p}}\right) \hookrightarrow C^{\{k\}}([0, T] ; C(\bar{\Omega}))$ and

$$
\begin{aligned}
& \left\|u_{2}\right\|_{C\{k\}}\left([0, T] ; X_{A_{p}}\right) \\
& \leq \widehat{C}\left(\|h\|_{C}^{\{k\}, \alpha_{2}\left([0, T] ; X_{p}\right)}+\|h(0, \cdot)\|_{X_{A_{p}}}+\|L \varphi\|_{X_{A_{p}}}\right)+\|\varphi\|_{X_{A_{p}}} .
\end{aligned}
$$

Let us consider the function $\mathcal{G}$ given by (5.16). (Recall that there $v_{2}=w_{2}-$ $\eta u_{2}$.) Due the proved properties of $w_{2}$ and $u_{2}$ and the assumptions of the theorem, it holds $\mathcal{G} \in C(\bar{\Omega})$ and $\|\mathcal{G}\|_{C(\bar{\Omega})} \leq \widehat{C}$ RHS.

Now, let us provide an estimate for $\|f\|_{C(\bar{\Omega})}$ using the formulas (5.15) and (5.17). Since $1 / g(T, \cdot) \in C(\bar{\Omega})$ and $v_{1}[\cdot](T, \cdot) \in \mathcal{L}\left(X_{p}, X_{A_{p}}\right)$, we have

$$
\begin{aligned}
& \|f\|_{C(\bar{\Omega})} \leq\|\mathcal{F} f\|_{C(\bar{\Omega})}+\|\mathcal{G}\|_{C(\bar{\Omega})} \leq \widehat{C}\left\|v_{1}[f](T, \cdot)\right\|_{C(\bar{\Omega})}+\|\mathcal{G}\|_{C(\bar{\Omega})} \\
& \leq \widehat{C}\left\|v_{1}[f](T, \cdot)\right\|_{X_{A_{p}}}+\|\mathcal{G}\|_{C(\bar{\Omega})} \leq \widehat{C}\|f\|_{X_{p}}+\|\mathcal{G}\|_{C(\bar{\Omega})} .
\end{aligned}
$$

Since $(I-\mathcal{F})$ is invertible in $X_{p}$, the estimate holds

$$
\|f\|_{X_{p}} \leq\left\|(I-\mathcal{F})^{-1}\right\|_{\mathcal{L}\left(X_{p}\right)}\|\mathcal{G}\|_{X_{p}} \leq \widehat{C}\|\mathcal{G}\|_{C(\bar{\Omega})}
$$

Thus, we obtain

$$
\|f\|_{C(\bar{\Omega})} \leq \widehat{C} \mathrm{RHS}
$$

Finally, let us derive an estimate for $u$ and finish the proof of the first part of the theorem. We have $u=u_{1}+u_{2}$, where $u_{1}=M * w_{1}, w_{1}=D_{t}^{\{k\}} u_{1}$ and $w_{1}$ solves the problem

$$
D_{t}^{\{k\}} w_{1}=A_{p} w_{1}+f D_{t}^{\{k\}} g, \quad w_{1}(0, \cdot)=0 .
$$

Since $f D_{t}^{\{k\}} g \in C_{0}^{\alpha^{\prime}}\left([0, T] ; X_{p}\right)$, Lemma 3 implies $w_{1} \in C_{0}^{\alpha^{\prime}}\left([0, T] ; X_{A_{p}}\right)$ and $\left\|u_{1}\right\|_{C_{0}^{\{k\}, \alpha^{\prime}}\left([0, T] ; X_{A_{p}}\right)}=\left\|w_{1}\right\|_{C_{0}^{\alpha^{\prime}}\left([0, T] ; X_{A_{p}}\right)} \leq \widehat{C}\|f\|_{C(\bar{\Omega})}\left\|D_{t}^{\{k\}} g\right\|_{C_{0}^{\alpha^{\prime}}\left([0, T] ; X_{p}\right)}$. Using here (5.23) we have

$$
\left\|u_{1}\right\|_{C_{0}^{\{k\}, \alpha^{\prime}}\left([0, T] ; X_{A_{p}}\right)} \leq \widehat{C} \mathrm{RHS} .
$$

From (5.22) and (5.24) we obtain for $u=u_{1}+u_{2}$ the estimate

$$
\|u\|_{C\{k\}\left([0, T] ; X_{A_{p}}\right)} \leq \widehat{C} \mathrm{RHS} .
$$

It remains to estimate $L u$ in the space $C([0, T] ; C(\bar{\Omega}))$. Using $(5.25)$ we deduce

$$
\left\|D_{t}^{\{k\}}(u-\varphi)\right\|_{C([0, T] ; C(\bar{\Omega}))} \leq \widehat{C}\left\|D_{t}^{\{k\}}(u-\varphi)\right\|_{C\left([0, T] ; X_{A_{p}}\right)} \leq \widehat{C} \mathrm{RHS} .
$$


From the expression $L u=D_{t}^{\{k\}}(u-\varphi)-f g-h$ due to the proved estimates for $D_{t}^{\{k\}}(u-\varphi)$ and $f$ we obtain

$$
\|L u\|_{C([0, T] ; C(\bar{\Omega}))} \leq \widehat{C} \mathrm{RHS} .
$$

Summing up, (5.23), (5.25) and (5.26) imply (5.19).

Now let us focus on the second part of this theorem that is concerned with the particular case $\varphi=h(0, \cdot)=D_{t}^{\{k\}} h(0, \cdot)=0$. Then RHS reduces to the expression in brackets at the right-hand side of (5.20). Lemma 3 implies that the function $w_{2}$ which solves $(5.21)$ belongs the space $C_{0}^{\alpha^{\prime}}\left([0, T] ; X_{A_{p}}\right)$, the function $u_{2}=M * w_{2}$ belongs to $C_{0}^{\{k\}, \alpha^{\prime}}\left([0, T] ; X_{A_{p}}\right)$ and $\left\|u_{2}\right\|_{C_{0}^{\{k\}, \alpha^{\prime}}{ }_{\left([0, T] ; X_{A_{p}}\right)} \leq} \leq$ $\widehat{C}\|h\|_{C_{0}^{\{k\}, \alpha_{2}}\left([0, T] ; X_{p}\right)}$. This relation by $u=u_{1}+u_{2}$ and the estimates (5.23), (5.24) and (5.26) implies (5.20).

Provided the assumptions of Theorem 3 hold and $\mathcal{B}=I$, an explicit expression of the unknown function $f$ at the boundary can be derived. Namely, setting $t=T$ and $x \in \partial \Omega$ in (2.6) and taking the relations $F=f g+h$ and $u(T, \cdot)=\psi$ into account we obtain $f(x)=-\frac{1}{g(T, x)}[L \psi(x)+h(T, x)], \quad x \in \partial \Omega$.

\section{Results on IP2}

In the context of IP2 let us introduce the following sets for the coefficient $r$ :

$$
\mathcal{K}_{R}=\{r \in C(\bar{\Omega}): r(x) \leq R, x \in \bar{\Omega}\}, \quad \text { where } R \in \mathbb{R} .
$$

Theorem 4. Let $R$ be some real number and IP2 have two solutions $(r, u)$, $\left(r_{1}, u_{1}\right)$, such that

$$
\begin{aligned}
& r \in C(\bar{\Omega}), \quad r_{1} \in \mathcal{K}_{R}, \quad u, u_{1} \in C_{0}^{\{k\}}\left([0, T] ; L_{1}(\Omega)\right) \bigcap C_{0}\left([0, T] ; W_{1}^{2}(\Omega)\right), \\
& u_{1}-u \in C_{0}^{\{k\}}([0, T] ; C(\bar{\Omega})) \bigcap C_{0}\left([0, T] ; W_{p}^{2}(\Omega)\right)
\end{aligned}
$$

for some $p>1$ and the function $U=u+b$ (and $M$ ) satisfy one of the following assumptions:

(A4) $U \in C_{0}^{1+\alpha_{1}}([0, T] ; C(\bar{\Omega}))$ for some $0<\alpha_{1}<1$;

(A5) $U \in C_{0}^{\{k\}, \alpha_{1}}([0, T] ; C(\bar{\Omega}))$ and $M(t) \geq c t^{\gamma-1}, t \in(0, T)$ for some $c \in \mathbb{R}_{+}$, $0<\gamma<\alpha_{1}<1$

(A6) $U \in C_{0}^{\{k\}, \alpha_{1}-\beta}([0, T] ; C(\bar{\Omega}))$ and $c_{1} t^{\gamma-1} \leq M(t) \leq c_{2} t^{\beta-1},\left|M^{\prime}(t)\right| \leq$ $c_{3} t^{\beta-2}, t \in(0, T)$, for some $c_{1}, c_{2}, c_{3} \in \mathbb{R}_{+}, 0<\beta \leq \gamma<\alpha_{1}<1$.

Additionally, we assume that

$$
\begin{aligned}
& U \geq 0, \quad D_{t}^{\{k\}} U-R U \geq 0, \\
& \text { a.e. } x \in \Omega, \quad \exists t_{x} \in(0, T]: U\left(t_{x}, x\right)>0 .
\end{aligned}
$$

In case $\mathcal{B}=I$ we also assume that $\forall x \in \partial \Omega$, either $U(T, x)>0$ or $U(\cdot, x)=0$. Then $\left(r_{1}, u_{1}\right)=(r, u)$. 
Proof. The difference $(\hat{r}, \hat{u})=\left(r_{1}-r, u_{1}-u\right) \in C(\bar{\Omega}) \times\left(C_{0}^{\{k\}}([0, T] ; C(\bar{\Omega}))\right.$ $\left.\bigcap C_{0}\left([0, T] ; W_{p}^{2}(\Omega)\right)\right)$ solves the problem

$$
\begin{aligned}
& D_{t}^{\{k\}} \hat{u}(t, x)=\left(L_{1}+r_{1}\right) \hat{u}(t, x)+U(t, x) \hat{r}(x), \quad x \in \Omega, t \in(0, T), \\
& \hat{u}(0, x)=0, \quad x \in \Omega, \quad \mathcal{B} \hat{u}(t, x)=0, \quad x \in \partial \Omega, t \in(0, T), \\
& \hat{u}(T, x)=0, \quad x \in \Omega .
\end{aligned}
$$

The inequalities (6.1) imply that $D_{t}^{\{k\}} U-R_{r} U \geq 0$, where $R_{r}:=\max _{x \in \bar{\Omega}} r_{1}(x) \leq$ $R$. Consequently, the assumptions of Theorem 1 are satisfied for the problem (6.2) and we obtain $\hat{r}=0, \hat{u}=0$.

Let us formulate a problem that contains approximate data:

$$
\begin{aligned}
& D_{t}^{\{k\}}(\tilde{u}-\tilde{\varphi})(t, x)=L_{1} \tilde{u}(t, x)+\tilde{r}(x)(\tilde{u}+\tilde{b})(t, x)+\tilde{F}_{1}(t, x), \quad x \in \Omega, t \in(0, T), \\
& \tilde{u}(0, x)=0, \quad x \in \Omega, \quad \mathcal{B} \tilde{u}(t, x)=0, \quad x \in \partial \Omega, t \in(0, T), \\
& \tilde{u}(T, x)=\tilde{\psi}, \quad x \in \Omega .
\end{aligned}
$$

We are going to prove an existence and approximation theorem for this problem in case its data vector $\widetilde{D}=\left(\tilde{b}, \tilde{F}_{1}, \tilde{\psi}\right)$ is close to the data vector $D=\left(b, F_{1}, \psi\right)$ of the exact problem IP2.

Theorem 5. Assume that $R \in \mathbb{R}$ and IP2 has a solution $(r, u) \in \mathcal{K}_{R}$ $\times C_{0}^{\{k\}}\left([0, T] ; L_{1}(\Omega)\right) \bigcap C_{0}\left([0, T] ; W_{1}^{2}(\Omega)\right)$ such that $U=u+b$ (and $\left.M\right)$ satisfy one of the assumptions (A4)-(A6), the inequalities (6.1) and $U(T, x)>0$, $x \in \bar{\Omega}$. Then the following statements are valid.

(i) Let $p \in\{0\} \bigcup\left(\frac{n}{2}, \infty\right), \alpha_{2} \in(0,1)$. There exist constants $\delta_{1}>0$ and $K_{1}>0$ depending on $M, L_{1}, r, U, p, \alpha_{2}$ such that if

$$
\tilde{D}-D \in \mathcal{D}_{1}=C_{0}^{\alpha_{2}}\left([0, T] ; C_{(p)}(\bar{\Omega})\right) \times C_{0}^{\alpha_{2}}\left([0, T] ; X_{p}\right) \times X_{A_{p}}
$$

and $\|\tilde{D}-D\|_{\mathcal{D}_{1}} \leq \delta_{1}$, where $C_{(p)}(\bar{\Omega})=\left\{\begin{array}{ll}C(\bar{\Omega}), & \text { in case } p \in\left(\frac{n}{2}, \infty\right), \\ X_{0}, & \text { in case } p=0,\end{array}\right.$ then problem (6.3) has a unique solution in the set

$$
\begin{aligned}
& \left\{(\tilde{r}, \tilde{u}):(\tilde{r}-r, \tilde{u}-u) \in \mathcal{X}_{1}:=X_{p} \times\left(C_{0}^{\{k\}, \alpha}\left([0, T] ; X_{p}\right) \bigcap C_{0}^{\alpha}\left([0, T] ; X_{A_{p}}\right)\right),\right. \\
& \left.\|(\tilde{r}-r, \tilde{u}-u)\|_{\mathcal{X}_{1}} \leq K_{1}\|\tilde{D}-D\|_{\mathcal{D}_{1}}\right\}
\end{aligned}
$$

where $\alpha= \begin{cases}\alpha_{2}, & \text { in case (A4), } \\ \min \left\{\alpha_{1}, \alpha_{2}\right\}, & \text { in cases (A5), (A6). }\end{cases}$

(ii) Let $p \in\left(\frac{n}{2}, \infty\right), \alpha_{2} \in(0,1)$. There exist constants $\delta_{2}>0$ and $K_{2}>0$ depending on $M, L_{1}, r, U, p, \alpha_{2}$ such that if

$$
\tilde{D}-D \in \mathcal{D}_{2}=\left(C_{0}^{\{k\}, \alpha_{2}}\left([0, T] ; X_{p}\right) \bigcap C_{0}^{\alpha_{2}}([0, T] ; C(\bar{\Omega}))\right)^{2} \times Y_{p}
$$


and $\|\tilde{D}-D\|_{\mathcal{D}_{2}} \leq \delta_{2}$ where $Y_{p}=\left\{\psi: \psi \in X_{A_{p}}, L \psi \in C(\bar{\Omega})\right\}$, then the problem (6.3) has a unique solution in the set

$$
\left\{(\tilde{r}, \tilde{u}):(\tilde{r}-r, \tilde{u}-u) \in \mathcal{X}_{2}:=C(\bar{\Omega}) \times \mathcal{U}_{p, \alpha^{\prime}},\|(\tilde{r}-r, \tilde{u}-u)\|_{\mathcal{X}_{2}} \leq K_{2}\|\tilde{D}-D\|_{\mathcal{D}_{2}}\right\}
$$

where $\mathcal{U}_{p, \alpha^{\prime}}=\left\{v \in C_{0}^{\{k\}, \alpha^{\prime}}\left([0, T] ; X_{A_{p}}\right): L v \in C_{0}([0, T] ; C(\bar{\Omega}))\right\}, \alpha^{\prime}=$ $\min \left\{\hat{\alpha} ; \alpha_{2}\right\}$ and $\hat{\alpha}= \begin{cases}\alpha_{1}, & \text { in cases }(\mathrm{A} 4),(\mathrm{A} 5), \\ \alpha_{1}-\beta, & \text { in case }(\mathrm{A} 6) \text {. }\end{cases}$

We mention that in this theorem, the operator $A_{p}$ and the space $X_{A_{p}}$ defined on the basis of $L=L_{1}+r I$ depend on the component $r$ of the solution of the exact problem IP2.

Proof. Let us denote the difference $(\hat{r}, \hat{u})=(\tilde{r}-r, \tilde{u}-u)$. Then the problem for the pair $(\hat{r}, \hat{u})$ reads

$$
\begin{aligned}
& D_{t}^{\{k\}} \hat{u}=\left(L_{1}+r\right) \hat{u}+\hat{r}(u+b)+\left[\hat{r} \hat{u}+\tilde{F}_{1}-F_{1}+(\hat{r}+r)(\tilde{b}-b)\right], \\
& \hat{u}(0, \cdot)=0,\left.\quad \mathcal{B} \hat{u}\right|_{\partial \Omega}=0, \quad \hat{u}(T, \cdot)=\tilde{\psi}-\psi .
\end{aligned}
$$

This problem can be treated as IP1 with $f=\hat{r}, g=u+b, h=\hat{r} \hat{u}+\tilde{F}_{1}-F_{1}+$ $(\hat{r}+r)(\tilde{b}-b)$. Therefore, applying the solution operator of IP1 $\mathcal{A}$ to $(6.4)$, it is reduced to the operator equation

$$
(\hat{r}, \hat{u})=\mathcal{F}_{2}(\hat{r}, \hat{u})
$$

where $\mathcal{F}_{2}(\hat{r}, \hat{u})=\mathcal{A}\left(\hat{r} \hat{u}+\tilde{F}_{1}-F_{1}+(\hat{r}+r)(\tilde{b}-b), 0, \tilde{\psi}-\psi\right)$.

We are going to show that $\mathcal{F}_{2}$ is a contraction in a ball $\|(\hat{r}, \hat{u})\|_{\mathcal{X}_{1}} \leq \rho$ with a suitable chosen $\rho>0$. Firstly, we have to prove that this ball remains invariant with respect to the operator $\mathcal{F}_{2}$. Let $\|(\hat{r}, \hat{u})\|_{\mathcal{X}_{1}} \leq \rho$. According to (5.10),

$$
\left\|\mathcal{F}_{2}(\hat{r}, \hat{u})\right\|_{\mathcal{X}_{1}} \leq C_{6}\left(\|\tilde{\psi}-\psi\|_{X_{A_{p}}}+\left\|\hat{r} \hat{u}+\tilde{F}_{1}-F_{1}+(\hat{r}+r)(\tilde{b}-b)\right\|_{C_{0}^{\alpha_{2}}\left([0, T] ; X_{p}\right)}\right) .
$$

Let $c_{p}$ be an embedding constant such that $\|w\|_{C(\bar{\Omega})} \leq c_{p}\|w\|_{X_{A_{p}}}$. Then

$$
\|\hat{r} \hat{u}\|_{C_{0}^{\alpha_{2}\left([0, T] ; X_{p}\right)}} \leq\|\hat{r}\|_{X_{p}}\|\hat{u}\|_{C_{0}^{\alpha}([0, T] ; C(\bar{\Omega}))} \leq\|\hat{r}\|_{X_{p}} c_{p}\|\hat{u}\|_{C_{0}^{\alpha}\left([0, T] ; X_{A_{p}}\right)} \leq c_{p} \rho^{2} .
$$

Therefore,

$$
\begin{aligned}
& \left\|\mathcal{F}_{2}(\hat{r}, \hat{u})\right\|_{\mathcal{X}_{1}} \leq C_{6}\left(\|\tilde{\psi}-\psi\|_{X_{A_{p}}}+c_{p} \rho^{2}+\left\|\tilde{F}_{1}-F_{1}\right\|_{C_{0}^{\alpha_{2}}\left([0, T] ; X_{p}\right)}\right. \\
& \left.+\left(\rho+R_{1}\right)\|\tilde{b}-b\|_{C_{0}^{\alpha_{2}}\left([0, T] ; C_{(p)}(\bar{\Omega})\right)}\right) \leq C_{6}\left(c_{p} \rho^{2}+\left(\rho+1+R_{1}\right)\|\tilde{D}-D\|_{\mathcal{D}_{1}}\right)
\end{aligned}
$$

where $R_{1}=\|r\|_{X_{p}}$ in case $p \in\left(\frac{n}{2}, \infty\right)$ and $R_{1}=\|r\|_{C(\bar{\Omega})}$ in case $p=0$. Now let us take $\rho=K_{1}\|\tilde{D}-D\|_{\mathcal{D}_{1}}$ with a constant $K_{1}$. Then

$$
\left\|\mathcal{F}_{2}(\hat{r}, \hat{u})\right\|_{\mathcal{X}_{1}} \leq C_{6}\left(\left(c_{p} K_{1}^{2}+K_{1}\right)\|\tilde{D}-D\|_{\mathcal{D}_{1}}+1+R_{1}\right)\|\tilde{D}-D\|_{\mathcal{D}_{1}} .
$$


In case $\|\tilde{D}-D\|_{\mathcal{D}_{1}} \leq \delta_{1}$ we have

$$
\left\|\mathcal{F}_{2}(\hat{r}, \hat{u})\right\|_{\mathcal{X}_{1}} \leq C_{6}\left(\left(c_{p} K_{1}^{2}+K_{1}\right) \delta_{1}+1+R_{1}\right)\|\tilde{D}-D\|_{\mathcal{D}_{1}} .
$$

Let us define the constants as follows: $K_{1}=C_{6}\left(2+R_{1}\right), \delta_{1}=\frac{1}{c_{p} K_{1}^{2}+K_{1}}$. Then $\left\|\mathcal{F}_{2}(\hat{r}, \hat{u})\right\|_{\mathcal{X}_{1}} \leq K_{1}\|\tilde{D}-D\|_{\mathcal{D}_{1}}$. Consequently, for $\|(\hat{r}, \hat{u})\|_{\mathcal{X}_{1}} \leq \rho$ we have $\left\|\mathcal{F}_{2}(\hat{r}, \hat{u})\right\|_{\mathcal{X}_{1}} \leq \rho$.

Secondly, inside the set $\|(\hat{r}, \hat{u})\|_{\mathcal{X}_{1}} \leq \rho=K_{1}\|\tilde{D}-D\|_{\mathcal{D}_{1}}$ let us consider the difference of $\mathcal{F}_{2}$ at $\left(\hat{r}_{1}, \hat{u}_{2}\right)$ and $\left(\hat{r}_{2}, \hat{u}_{2}\right)$. Assuming $\|\tilde{D}-D\|_{\mathcal{D}_{1}} \leq \delta_{1}$, we deduce the estimate

$$
\begin{aligned}
& \left\|\mathcal{F}_{2}\left(\hat{r}_{1}, \hat{u}_{1}\right)-\mathcal{F}_{2}\left(\hat{r}_{2}, \hat{u}_{2}\right)\right\|_{\mathcal{X}_{1}} \leq\|\mathcal{A}\| \|\left(\hat{r}_{1}-\hat{r}_{2}\right) \hat{u}_{1}+\hat{r}_{2}\left(\hat{u}_{1}-\hat{u}_{2}\right) \\
& \quad+\left(\hat{r}_{1}-\hat{r}_{2}\right)(\tilde{b}-b) \|_{C_{0}^{\alpha_{2}}\left([0, T] ; X_{p}\right)} \leq C_{6}\left(c_{p} \rho\left\|\hat{r}_{1}-\hat{r}_{2}\right\|_{X_{p}}\right. \\
& \left.\quad+c_{p} \rho\left\|\hat{u}_{1}-\hat{u}_{2}\right\|_{C_{0}^{\alpha}\left([0, T] ; X_{A_{p}}\right)}+\delta_{1}\left\|\hat{r}_{1}-\hat{r}_{2}\right\|_{X_{p}}\right) \leq C_{6}\left(c_{p} K_{1} \delta_{1}+\delta_{1}\right) \\
& \quad \times\left\|\left(\hat{r}_{1}-\hat{r}_{2}, \hat{u}_{1}-\hat{u}_{2}\right)\right\|_{\mathcal{X}_{1}}=\frac{1}{\left(2+R_{1}\right)}\left\|\left(\hat{r}_{1}-\hat{r}_{2}, \hat{u}_{1}-\hat{u}_{2}\right)\right\|_{\mathcal{X}_{1}} .
\end{aligned}
$$

It shows that the operator $\mathcal{F}_{2}$ is a contraction in the ball $\|(\hat{r}, \hat{u})\|_{\mathcal{X}_{1}} \leq \rho$. According to the Banach fixed point theorem there exists a unique solution to the equation (6.5) in that ball. This proves the assertion (i).

(ii) The proof of (ii) repeats the proof of (i) with appropriate changes of spaces and norms. For $\mathcal{A}$, the estimate (5.20) is used instead of (5.10).

Remark 1. In case the data of (6.3) are close to data of a process without reaction (i.e. $r=0$ ), Theorem 5 implies the existence of the reaction coefficient $\tilde{r}$ in small.

Remark 2. Supposing the existence of a solution $(r, u)$ of IP2, we ask: what are sufficient conditions on the data that guarantee the validity of inequality-type conditions (6.1) and $U(T, x)>0, x \in \bar{\Omega}$ in Theorems 4, 5? To answer this question, we return to the problem $(2.3)$ for $U$ and set there $\Phi=H(0, \cdot)=0$. Let us suppose that $U$ is sufficiently smooth. Then constructing a corresponding problem for $D_{t}^{\{k\}} U-R U$ and assuming $D_{t}^{\{k\}} H-R H \geq 0,\left.\left(D_{t}^{\{k\}} \mathcal{B} b-R \mathcal{B} b\right)\right|_{\partial \Omega} \geq 0$, Lemma 4 (i) implies the inequality $D_{t}^{\{k\}} U-R U \geq 0$. Next, we consider the conditions $U \geq 0$ and $U(T, x)>0, x \in \bar{\Omega}$. Let us assume that

$$
\begin{aligned}
& \exists \mu \in C[0, T], \mu \geq 0, \mu \neq 0, \mu \text { - nondecreasing : } \\
& H(t, x) \geq \mu(t), x \in \bar{\Omega}, t \in[0, T], \quad \mathcal{B} b(t, x) \geq \mu(t), x \in \partial \Omega, t \in[0, T] .
\end{aligned}
$$

Define $V=U-\delta 1 * \mu$ with $\delta>0$. The function $V$ solves the problem

$$
D_{t}^{\{k\}} V=L V+H_{1}, \quad V(0, \cdot)=0,\left.\quad \mathcal{B}(V-(b-\delta 1 * \mu))\right|_{\partial \Omega}=0,
$$

where $H_{1}=H+\delta\left(r 1 * \mu-D_{t}^{\{k\}} 1 * \mu\right)$. Since $D_{t}^{\{k\}} 1 * \mu=k * \mu$, we get that for sufficiently small $\delta$,

$$
H_{1}(t, x) \geq \mu(t)\left[1-\delta\left(\max _{x \in \bar{\Omega}} r(x) T+\|k\|_{L_{1}(0, T)}\right)\right] \geq 0, \quad t \in[0, T], \quad x \in \Omega
$$


and $\left.\mathcal{B} V\right|_{\partial \Omega}=\left.\mathcal{B}(b-\delta 1 * \mu)\right|_{\partial \Omega} \geq 0$. Lemma 4 (i) yields $V \geq 0$. Thus, $U=$ $V+\delta 1 * \mu \geq 0$ and $U(T, x)=V(T, x)+\delta \int_{0}^{T} \mu(\tau) d \tau>0, x \in \bar{\Omega}$.

At the end of this section, we make some general remarks. We applied results on IP1 to analyze IP2. In a similar manner, results on IP1 can be applied to study inverse problems to determine other coefficients of $L$, too.

The basic set of assumptions (A1)-(A3) for $g$ involves the restriction $g(0, \cdot)=0$. This is due to the fact that in case $g(0, \cdot) \neq 0$ we cannot ensure sufficient regularity of $u$ to apply the positivity principle in the proof of Theorem 1 . In IP2, the function $u+b=U$ works as $g$. For that reason, we consider the case $\Phi=U(0, \cdot)=0$ in IP2.

In the beginning of the proof of Lemma 4 we showed that the direct problem with $r>0$ can be reduced to a problem with $r \leq 0$ by the change of unknown $\tilde{u}=e^{-\sigma t} u$, where $\sigma>0$. This suggests a possible exponential growth of $u$ and a related time limitation of the linear reaction model in case $r>0$. For bigger $T$, nonlinear reaction models are more relevant [6].

Solutions of IP1 and IP2 depend continuously on derivatives of the data of finite order. This means that these problems are moderately ill-posed. In case approximate data are given with errors, regularization procedures can be effectively applied (cf. e.g. [17] for IP1 with $g=g(t)$ ).

\section{Acknowledgements}

The research was supported by the Estonian Research Council, Grant PUT568. Authors thank the referee whose valuable comments led to the improvement of the paper.

\section{References}

[1] E. Bajlekova. Fractional evolution equations in Banach spaces. PhD thesis, Technische Universiteit Eindhoven, Eindhoven, 12001.

[2] E. Beretta and C. Cavaterra. Identifying a space dependent coefficient in a reaction-diffusion equation. Inverse Problems and Imaging, 5(2):285-296, 2011. https://doi.org/10.3934/ipi.2011.5.285.

[3] A.V. Chechkin, V.Yu. Gonchar, R. Gorenflo, N. Korabel and I.M. Sokolov. Generalized fractional diffusion equations for accelerating subdiffusion and truncated Lévy flights. Physical Review E, 78:021111, 2008. https://doi.org/10.1103/PhysRevE.78.021111.

[4] A.V. Chechkin, R. Gorenflo and I.M. Sokolov. Fractional diffusion in inhomogeneous media. J. Phys. A: Math. Gen., 38(42):679-684, 2005. https://doi.org/10.1088/0305-4470/38/42/L03.

[5] P. Clement and J. Prüss. Completely positive measures and Feller semigroups. Mathematische Annalen, 287(1):73-105, 1990. https://doi.org/10.1007/BF01446879.

[6] D. Frömberg. Reaction Kinetics under Anomalous Diffusion. PhD thesis, Humboldt-Universität zu Berlin, Berlin, 81981. 
[7] K.M. Furati, O.S. Iyiola and M. Kirane. An inverse problem for a generalized fractional diffusion. Applied Mathematics and Computation, 249:24-31, 2014. https://doi.org/10.1016/j.amc.2014.10.046.

[8] D. Gilbarg and N. Trudinger. Elliptic Partial Differential Equations of Second Order. Springer, Berlin, 1977. https://doi.org/10.1007/978-3-642-96379-7.

[9] G. Gripenberg. On Volterra equations of the first kind. Integral Equations and Operator Theory, 3(4):473-488, 1980. https://doi.org/10.1007/BF01702311.

[10] G. Gripenberg, S.-O. Londen and O. Staffans. Volterra Integral and Functional Equations. Cambridge University Press, Cambridge, 1990. https://doi.org/10.1017/CBO9780511662805.

[11] G.H. Hardy and J.E. Littlewood. Some properties of fractional integrals I. Mathematische Zeitschrift, 27:565-606, 1928. https://doi.org/10.1007/BF01171116.

[12] V. Isakov. Inverse Problems for Partial Differential Equations. Springer, New York, 2006.

[13] J. Janno and K. Kasemets. A positivity principle for parabolic integro-differential equations and inverse problems with final overdetermination. Inverse Problems and Imaging, 3(1):17-41, 2009. https://doi.org/10.3934/ipi.2009.3.17.

[14] J. Janno and K. Kasemets. Identification of a kernel in an evolutionary integral equation occurring in subdiffusion. Journal of Inverse and Ill-posed Problems, 25(6):777-798, 2017. https://doi.org/10.1515/jiip-2016-0082.

[15] J. Janno and K. Kasemets. Uniqueness for an inverse problem for a semilinear time-fractional diffusion equation. Inverse Problems and Imaging, 11(1):125149, 2017. https://doi.org/10.3934/ipi.2017007.

[16] K. Kasemets and J. Janno. Reconstruction of a source term in a parabolic integro-differential equation from final data. Mathematical Modelling and Analysis, 16(2):199-219, 2011. https://doi.org/10.3846/13926292.2011.578282.

[17] N. Kinash and J. Janno. Inverse problems for a perturbed time fractional diffusion equation with final overdetermination. Math. Meth. Appl. Sci., 41(5):19251943, 2018. https://doi.org/10.1002/mma.4719.

[18] M. Kirane, S.A. Malik and M.A. Al-Gwaiz. An inverse source problem for a two dimensional time fractional diffusion equation with nonlocal boundary conditions. Math. Meth. Appl. Sci., 36(9):1056-1069, 2013. https://doi.org/10.1002/mma.2661.

[19] A.N. Kochubei. General fractional calculus, evolution equations, and renewal processes. Integral Equations and Operator Theory, 71(4):583-600, 2011. https://doi.org/10.1007/s00020-011-1918-8.

[20] Y. Liu, M. Yamamoto and W. Rundell. Strong maximum principle for fractional diffusion equations and an application to an inverse source problem. Fractional Calculus and Applied Analysis, 19(4), 2016. https://doi.org/10.1515/fca-20160048 .

[21] A. Lopushansy and H. Lopushanska. Inverse source Cauchy problem for a time fractional diffusion-wave equation with distributions. Electronic Journal of Differential Equations, 2017(182):1-14, 2017.

[22] Y. Luchko. Maximum principle and its application for the time-fractional diffusion equations. Fractional Calculus and Applied Analysis, 14(1):110-124, 2011. https://doi.org/10.2478/s13540-011-0008-6. 
[23] Y. Luchko and M. Yamamoto. General time-fractional diffusion equation: Some uniqueness and existence results for the initial-boundary-value problems. Fractional Calculus and Applied Analysis, 19(3):676-695, 2016. https://doi.org/10.1515/fca-2016-0036.

[24] A. Lunardi. Analytic Semigroups and Optimal Regularity in Parabolic Problems. Birkhäuser, Basel, 1995.

[25] F. Mainardi, A. Mura, G. Pagnini and R. Gorenflo. Time-fractional diffusion of distributed order. Journal of Vibration and Control, 14(9-10):1267-1290, 2008. https://doi.org/10.1177/1077546307087452.

[26] D.G. Orlovsky. Parameter determination in a differential equation of fractional order with Riemann-Liouville fractional derivative in a Hilbert space. Journal of Siberian Federal University, 8(1):55-63, 2015. https://doi.org/10.17516/19971397-2015-8-1-55-63.

[27] Y. Povstenko. Fractional heat conduction and associated thermal stress. Journal of Thermal Stresses, 28(1):83-102, 2004. https://doi.org/10.1080/014957390523741.

[28] J. Prüss. Evolutionary Integral Equations and Applications. Birkhäuser Verlag, Basel, 1993. https://doi.org/10.1007/978-3-0348-8570-6.

[29] F. Sabzikar, M.M. Meerschaert and J. Chen. Tempered fractional calculus. Journal of Computational Physics, 293:14-28, 2015. https://doi.org/10.1016/j.jcp.2014.04.024.

[30] K. Sakamoto and M. Yamamoto. Inverse source problem with a final overdetermination for a fractional diffusion equation. Mathematical Control \& Related Fields, 1(4):509-518, 2011. https://doi.org/10.3934/mcrf.2011.1.509.

[31] I.M. Sokolov, A.V. Chechkin and J. Klafter. Distributed-order fractional kinetics. Acta Physica Polonica B, 35(47):1323-1341, 2004.

[32] S. Tatar and S. Ulusoy. An inverse source problem for a one-dimensional spacetime fractional diffusion equation. Applicable Analysis, 94(11):2233-2244, 2015. https://doi.org/10.1080/00036811.2014.979808.

[33] V. Vergara and R. Zacher. Stability, instability, and blowup for time fractional and other nonlocal in time semilinear subdiffusion equations. Journal of Evolution Equations, 17(1):599-626, 2017. https://doi.org/10.1007/s00028-016-03702 .

[34] R. Zacher. Weak solutions of abstract evolutionary integro-differential equations in Hilbert spaces. Funkcialaj Ekvacioj, 52(1):1-18, 2009. https://doi.org/10.1619/fesi.52.1.

\section{Appendix: Proofs of Lemmas 1, 2 and 5}

Proof of Lemma 1. Theorems 3 and 4 of [9] guarantee that $k$ is nonnegative, nonincreasing and convex. Convexity implies the continuity of $k$. From the equation $M * k=1$ we easily deduce $\lim _{t \rightarrow 0^{+}} k(t)=+\infty$, because in the opposite case $k$ is bounded from which it follows that $\lim _{t \rightarrow 0^{+}}(M * k)(t)=0$.

Let us prove $k>0$. Suppose that it is not true. Then in view of proved properties of $k, \exists t_{0}: k(t)>0, t<t_{0}$ and $k(t)=0, t>t_{0}$. For $t>t_{0}$ from $M * k=1$ we get $\int_{0}^{t_{0}} M(t-\tau) k(\tau) d \tau=1$. Therefore, $\int_{0}^{t_{0}} M^{\prime}(t-\tau) k(\tau) d \tau=0$. 
The last equality contradicts to the assumptions $k(t)>0, t \in\left(0, t_{0}\right)$ and $M^{\prime}<0$. Thus, $k>0$.

Finally, let us prove (3.3) Let us choose some $t_{3}>0$. Since $\lim _{t \rightarrow 0^{+}} k(t)=+\infty$, there exists an interval $(0, \delta), \delta<t_{3}$, such that $k(t)>k\left(t_{3}\right)$ for $t \in(0, \delta)$. Suppose that (3.3) is not true. Then we can find two points $t_{1}<t_{2}$ in $(0, \delta)$ so that $k\left(t_{1}\right)=k\left(t_{2}\right)$. Consequently, for $t_{1}<t_{2}<t_{3}$ we have $k\left(t_{1}\right)=k\left(t_{2}\right)>$ $k\left(t_{3}\right)$. Obviously, it contradicts to the convexity of $k$. Therefore, (3.3) is valid.

Proof of Lemma 2 is similar to proof of Theorem 14 in [11] that is concerned with the case $M(t)=\frac{t^{\beta-1}}{\Gamma(\beta)}$. Let $z \in C_{0}^{\alpha-\beta}([0, T] ; X)$. Then $\|M * z(t)\|_{X} \leq$ const $t^{\beta-1} * t^{\alpha-\beta}=O\left(t^{\alpha}\right)$. Secondly,

$$
(M * z)(t)-(M * z)(t-h)=J_{1}+J_{2}+J_{3},
$$

where

$$
\begin{aligned}
& J_{1}=z(t) \int_{t-h}^{t} M(\tau) d \tau, \quad J_{2}=-\int_{0}^{h}[z(t)-z(t-\tau) M(\tau) d \tau, \\
& J_{3}=-\int_{h}^{t}[z(t)-z(t-\tau)] \int_{\tau-h}^{\tau} M^{\prime}(s) d s d \tau .
\end{aligned}
$$

Immediately, $\left\|J_{2}\right\|_{X} \leq$ const $\int_{0}^{h} \tau^{\alpha-\beta} \tau^{\beta-1} d \tau=O\left(h^{\alpha}\right)$. Moreover,

$$
\begin{aligned}
& \left\|J_{1}\right\|_{X} \leq \mathrm{const} t^{\alpha-\beta} \int_{t-h}^{t} \tau^{\beta-1}=\mathrm{const} t^{\alpha-\beta}\left[t^{\beta}-(t-h)^{\beta}\right] \\
& \left\|J_{3}\right\|_{X} \leq \mathrm{const} \int_{h}^{t} \tau^{\alpha-\beta} \int_{\tau-h}^{\tau} s^{\beta-2} d s d \tau=\mathrm{const} \int_{h}^{t} \tau^{\alpha-\beta}\left[(\tau-h)^{\beta-1}-\tau^{\beta-1}\right] d \tau .
\end{aligned}
$$

Further estimation of $J_{1}$ and $J_{3}$ can be performed exactly as in [11]. As a result, we get $\left\|J_{1}\right\|_{X},\left\|J_{3}\right\|_{X}=O\left(h^{\alpha}\right)$. This completes the proof.

Proof of Lemma 5. Firstly, we point out that the assumption $w \in W_{p}^{2}(\Omega), p>n$ implies $w \in C^{1}(\bar{\Omega})$. We will use maximum principles for elliptic equations in Sobolev spaces to prove the lemma. Let us consider the case $x^{*} \in \Omega$. Suppose that $L_{1} w\left(x^{*}\right)<0$. Then there exists a ball $B\left(x^{*}, \varepsilon\right) \subset \Omega$ and $\delta>0$ such that $L_{1} w(x) \leq-\delta<0$ for $x \in B\left(x^{*}, \varepsilon\right)$. Let us define the auxiliary function

$$
z(x)=\alpha\left|x-x^{*}\right|^{2} \text { with } \alpha>0
$$

such that $L_{1}(w+z) \leq 0$ in $B\left(x^{*}, \varepsilon\right)$. Since $w\left(x^{*}\right) \leq w(x)$ and $z\left(x^{*}\right)<z(x)$ for $x \in \partial B\left(x^{*}, \varepsilon\right)$, we get

$$
(w+z)\left(x^{*}\right)<(w+z)(x), \quad x \in \partial B\left(x^{*}, \varepsilon\right) .
$$

On the other hand, due to $L_{1}(w+z) \leq 0$ it follows from the Theorem 9.1 [8] that $\min _{x \in B\left(x^{*}, \varepsilon\right)}(w+z)(x)=\min _{x \in \partial B\left(x^{*}, \varepsilon\right)}(w+z)(x)$, that contradicts (7.2). Therefore, the supposition $L_{1} w\left(x^{*}\right)<0$ was wrong. 
Next let us consider the case $x^{*} \in \partial \Omega$. Again, suppose $L_{1} w\left(x^{*}\right)<0$. Then there exists $B\left(x^{*}, \varepsilon\right)$ and $\delta>0$ such that $L_{1} w(x) \leq-\delta<0$ for $x \in$ $B\left(x^{*}, \varepsilon\right) \bigcap \Omega$. Similarly to the previous case we define $z$ by $(7.1)$ so that $L_{1}(w+z) \leq 0$ in $B\left(x^{*}, \varepsilon\right) \cap \Omega$. Then $(w+z)\left(x^{*}\right)<(w+z)(x)$ for $x \in$ $B\left(x^{*}, \varepsilon\right) \bigcap \Omega$. Hence, Lemma 3.4 [8] is applicable and yields $\frac{\partial w}{\partial \nu}\left(x^{*}\right)=$ $\frac{\partial(w+z)}{\partial \nu}\left(x^{*}\right)<0$. That contradicts to $\frac{\partial}{\partial \nu} w\left(x^{*}\right) \geq 0$ following from the assumption $\frac{\partial}{\partial \omega} w\left(x^{*}\right) \geq 0$. Therefore, $L_{1} w\left(x^{*}\right) \geq 0$ holds. 